\title{
Millimeter Wave Small Cell Network Planning for Outdoor Line-of-Sight Coverage
}

\author{
by \\ Nima Palizban, B.Sc. \\ A thesis submitted to the \\ Faculty of Graduate and Postdoctoral Affairs \\ in partial fulfillment of the requirements for the degree of
}

Master of Applied Science in Electrical and Computer Engineering

Ottawa-Carleton Institute for Electrical and Computer Engineering

Department of Systems and Computer Engineering

Carleton University

Ottawa, Ontario

June, 2017

(C) Copyright

Nima Palizban, 2017 
The undersigned hereby recommends to the

Faculty of Graduate and Postdoctoral Affairs acceptance of the thesis

\title{
Millimeter Wave Small Cell Network Planning for Outdoor Line-of-Sight Coverage
}

\author{
submitted by Nima Palizban, B.Sc. \\ in partial fulfillment of the requirements for the degree of
}

Master of Applied Science in Electrical and Computer Engineering

Ottawa-Carleton Institute for Electrical and Computer Engineering

Department of Systems and Computer Engineering

Carleton University

June, 2017 


\section{Abstract}

The increasing spectrum demand drives next-generation wireless networks to use higher carrier frequencies. The millimeter wave band has orders of magnitude more bandwidth available and is considered as an important candidate for providing most of the capacity in a future heterogeneous network. Moreover, network densification is necessary for enhanced capacity and high-rate coverage. However, deploying a very large number of the base stations might be prohibitively costly. We develop an outdoor small cell millimeter wave network planner and minimize the number of base stations for line-of-sight coverage. This thesis consists of two proposals.

First, we estimate the base station densities required for small cell line-of-sight millimeter wave networks. To find the answer, we develop an automatic outdoor network planner by first, placing many candidates on the map and then, selecting a subset of candidates to minimize the total number of base stations and ensure a high percentage outdoor coverage. We run our simulations over two large dense urban areas. Our results show the effects of city layout on the millimeter wave networks.

Second, we design a wireless backhaul network planner to further reduce the deployment cost by reducing the number of wired connections in the network. We integrate millimeter wave line-of-sight wireless backhauling into our network planner

and minimize the number of wired connections required. The results indicate the advantages of wireless backhauling to reduce the small cell deployment cost. 


\section{Acknowledgments}

I would like to first thank my supervisors Dr. Sebastian Szyszkowicz and Prof. Halim Yanikomeroglu for their kind support during this thesis. Special thanks to Dr. Sebastian Szyszkowicz for his effective presence, encouragement, and guidance throughout the course of my Master's program. Also, I would like to thank Dr. Tamer Beitelmal regarding his kind advice, proofreadings, and the time he spent with me discussing technical issues.

I would also like to acknowledge the National Sciences and Engineering Research Council (NSERC) of Canada, the TELUS Corporation, and the Queen Elizabeth II Scholarship in Science and Technology for their financial support during the time of this work. I was really grateful to be a part of Carleton University during this work, as I learned, practiced, and enjoyed my presence in this community.

Finally and most importantly, I am thankful to my parents for their unconditional support and encouragement on my academic advancement during my entire life. And my wife for her patience, warm respect, and kind support. 


\section{Table of Contents}

Abstract $\quad$ iii

Acknowledgments $\quad$ iv

Table of Contents $\quad$ v

List of Tables $\quad$ viii

List of Figures $\quad$ ix

Nomenclature $\quad$ Xv

1 Introduction 1

1.1 Design Overview . . . . . . . . . . . . . . . . 4

1.2 Contributions and Novelty . . . . . . . . . . . . . . . 5

1.3 Many Maps . . . . . . . . . . . . . . . . . . . . . 7

1.4 Organization of the Thesis and Publications . . . . . . . . . 8

1.4.1 Organization .................. 8

1.4.2 Publications ................... 9

2 Literature Review and Problem Statement 10

2.1 Next Generation Wireless Networks Overview . . . . . . . . . . . . . 10

2.2 Millimeter Wave Propagation . . . . . . . . . . . . . . . . . 12 
2.3 Wireless Backhauling . . . . . . . . . . . . . . . . . . . . 14

2.4 Network Planning . . . . . . . . . . . . . . . . . . 16

2.5 Blockage Modelling and Real Maps . . . . . . . . . . . . . . . 17

2.6 Proposed System Design . . . . . . . . . . . . . . . . . . 19

3 Related Topics $\quad 23$

3.1 Visibility Polygon . . . . . . . . . . . . . . . . . . . . 24

3.2 Art Gallery Problem . . . . . . . . . . . . . . . . 24

3.2.1 Art Gallery Problem with Cooperative Guards . . . . . . . . . 27

3.2.2 Art Gallery Problem in Practice . . . . . . . . . . . . . . 28

3.3 Graph Connectivity . . . . . . . . . . . . . . . . 28

3.4 Connected Subgraph Problem . . . . . . . . . . . . . . . . . 32

3.5 Integer Linear Programming Terminology . . . . . . . . . . . . . . . . 35

4 Millimeter Wave Access Network Planning 38

4.1 Introduction . . . . . . . . . . . . . . . . . 38

4.2 Problem Statement . . . . . . . . . . . . . . . . . . 40

4.3 Candidate Location Placement . . . . . . . . . . . . . . . . . 41

4.4 Maximizing the Coverage . . . . . . . . . . . . . . . . . . . . . 42

4.4.1 Greedy Addition . . . . . . . . . . . . . . . 45

4.4.2 Binary Integer Linear Programming . . . . . . . . . . . . . . . 46

4.4.3 Upper Bound on Coverage . . . . . . . . . . . . . . . . . 47

4.5 Simulation and Results . . . . . . . . . . . . . . . . 48

4.5.1 Density of BSs for Different LOS Coverage Percentages . . . . 48

4.5.2 Probability Distribution of Cell's LOS Fraction . . . . . . . . 51

4.5.3 Noise and Interference Analysis in Millimeter Wave Networks 51 
5 Millimeter Wave Backhaul Network Planning

5.1 Introduction . . . . . . . . . . . . . . . . . . 59

5.2 System Model and Problem Statement . . . . . . . . . . . . . 61

5.2.1 Fresnel Zones . . . . . . . . . . . . . . . . . . . 61

5.2 .2 Engineering Setup . . . . . . . . . . . . . 62

5.3 Maximizing the Coverage with Connectivity . . . . . . . . . . . 64

5.3.1 Mixed Integer Linear Programming . . . . . . . . . . . . . . . 65

5.3.2 Greedy Approach . . . . . . . . . . . . . . . . . 68

5.3.3 Upper Bound on Coverage . . . . . . . . . . . . . . . . 68

5.4 Minimizing the Number of Gateways and Load Balancing . . . . . . . 69

5.5 Simulations and Results . . . . . . . . . . . . . 73

5.5.1 Planned Networks . . . . . . . . . . . . . . . . . 74

5.5.2 ABS and Gateway Densities . . . . . . . . . . . . . . . 74

5.5.3 Probability Distribution of Links Distance . . . . . . . . 78

5.5.4 Load Balancing . . . . . . . . . . . . . . . . . 78

6 Conclusion and Future Work 80

6.1 Conclusion . . . . . . . . . . . . . . . . . 80

6.2 Future Work . . . . . . . . . . . . . . . . . . . . . . . 82

$\begin{array}{lr}\text { List of References } & 84\end{array}$ 


\section{List of Tables}

2.1 Brief comparision of different wireless backhaul options. . . . . . . 15

4.1 Simulation Setup and Results Summary . . . . . . . . . . . . 55 


\section{List of Figures}

2.1 Different maps, Manhattan map on the top left, Paris on the top right, with two toy maps, Manhattan grid on the bottom left and random rectangles on the bottom right. It can be seen that different cities have different outdoor area shapes which affect the line-of-sight area from a base station. Bending streets in Paris and straight long streets in Manhattan affect the networks in different ways. Also, a random rectangle map might not be similar enough to dense urban areas, and would be a better fit for rural areas. . . . . . . . . . . . . .

2.2 Engineering setup. As LOS communication is preferred, mmWave backhauling can bring connection to areas not visible by the gateway.

3.1 Visibility Polygon, the center point is represented with blue, the yellow area is the visibility polygon associated to the center, and the green area is the coverage within $200 \mathrm{~m}$ cell radius. . . . . . . . . . . .

3.2 Upper bound on the number guards to cover a simple polygon art gallery. A coloring argument can be used to show that it is possible to guard the gallery with $\lfloor n / 3\rfloor$ guards, where $n$ is the number of vertices. Here, if we pick the green color, then 2 guards are enough which is equal to $\lfloor 7 / 3\rfloor$. The dashed lines show the triangulation of the gallery. . . 26

3.3 A five node connected graph. . . . . . . . . . . . . . . 
3.4 A network flow diagram. The flow conservation assumtion for node 1 implies $b_{1}+\left(x_{01}+x_{21}\right)-\left(x_{12}+x_{10}\right)=s_{1} \ldots \ldots \ldots$

4.1 An example of how adjacent buildings are merged together and holes inside them are removed to create a simple polygon. . . . . . . . .

4.2 Candidate location placement. The red lines are the shortest links between natural neighbour simple polygons. Candidate locations are found by searching each segment in the simple polygon for the point with largest line-of-sight coverage area. That is to say, each candidate location has a local maximum line-of-sight coverage. . . . . . . . . .

4.3 Result of candidate location placement algorithm with distributed points. To model the coverage, we distribute grid points on the map and create binary matrix $C$, where each candidate location takes one row in the matrix and each point one column. Coverage area of one example candidate location is indicated by yellow area. . . . . . . .

4.4 Manhattan grid map. It is designed to be similar to highly dense urban areas where we used $20 \mathrm{~m}, 150 \mathrm{~m}$, and $50 \mathrm{~m}$ for street width, building footprints' width and height, respectively. Candidate locations are indicated with blue circles, where we placed them on building corners as well as the middle of each side. Selected candidates are shown in red, where the result is find by running the binary integer linear

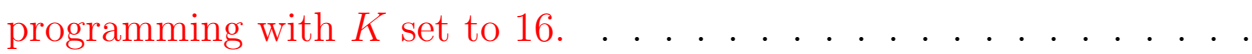


4.5 Base station densities required for different outdoor line-of-sight coverge fraction. The figure shows the simulation results for different urban areas, as well as the Manhattan grid map. we found 40, 50 and 60 base station per $\mathrm{km}^{2}$ densities to cover 90 percent of outdoor area. Also, the inner figure compares the differences between the greedy addition, binary integer linear programming and the bound. . . . . .

4.6 Cumulative density function of the line-of-sight fraction of a cell for different cell radiuses. When we consider $200 \mathrm{~m}$ cell radiuses, the probability of having more than 20 percent line of sight area in a cell drops to less than 10 percent. Also, the curves saturate at around 75 percent due to the fact that we are placing the base stations on wall corners, meaning that there is always 25 percent blocked by the mounted building. . . . . . . . . . . . . . . .

4.7 Number of times a certain area is covered, for 90 percent outdoor area coverage. A typical outdoor user is covered by about 1.4 base stations on average, meaning that the number of strong interferers (both lineof-sight and within $200 \mathrm{~m}$ ) is about 0.5 on average. There are almost never more than 3 strong interferers . . . . . . . . . . . .

4.8 Power distribution in planned maps. A large fraction of the outdoor area is covered with SINR of more than $20 \mathrm{~dB}$. The figure suggests that low SINR is mainly caused by interference, and the SINR curve is more similar to the SIR curve for small SINR values (less than $20 \mathrm{~dB}$ ), and it is more similar to the SNR curve for larger SINR values (more than $35 \mathrm{~dB}) . \ldots \ldots \ldots \ldots \ldots$ 
4.9 Result of the binary integer linear programming with 50 base stations per $\mathrm{km}^{2}$ in Manhattan Island area, with its corresponding spatial SINR distribution. White regions do not have a serving LOS link and are considered in outage. . . . . . . . . . . . . . . . .

5.1 Fresnel zones approximation. We approximate the Fresnel zones with a diagonal shap. The figure shows the first and 10th Fresnel zones in dark and light green, respectively. As can be seen, in millimeter wave frequencies, the maximum first Fresnel zone radius is much smaller than building dimensions. . . . . . . . . . . . . . . . .

5.2 Network planner steps. Candidate location placement is briefly described in Section 4.3. Access base station and selection and gateway placement algorithms are described in Section 5.3 and Section 5.4, re-

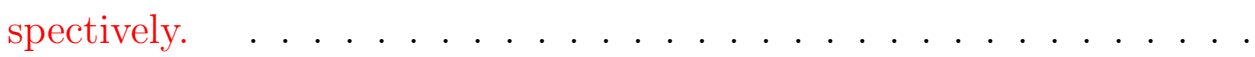

5.3 An example of the flow diagram in a three node network. The flow conservation assumption applies to all of the nodes in the graph, for $n_{1}$ it becoms as follows: $b_{1}+x_{01}+x_{21}-x_{10}-x_{12}=K s_{1}$, where $K$ is the number of candidate locations we want to select. . . . . . . . 
5.4 Result of maximum coverage problem with connectivity. Access base stations are shown with red circles, possible wireless backhaul links with black lines (representing the first Fresnel zone diamond approximation), and total LOS coverage area is shown with green. As can be seen, there are some places which are not covered, which is due to the fact that even when all candidate locations are selected there are some areas which are not covered. The places are found by running the algorithm in Section 5.3 over a $600 \mathrm{~m} \times 600 \mathrm{~m}$ area, with $K$ (number of access base stations) set to 20, and the total coverage is around 92 percent of the outdoor area. . . . . . . . . . . . . .

5.5 Minimizing the number of gateways. Result of the algorithm in Section 5.4 with the allowable number of hops set to 1 (single-hop network). Gateways are shown with purple pentagons, access base stations are shown with red circles, and the total line of sight coverage area is represented in green. In this particular map we need only 7 gateways to connect 20 base stations to the core network. . . . . . . . . .

5.6 Minimizing the number of gateways. Result of the algorithm in Section 5.4 with allowable number of hops set to 2 . We solve the optimization problem to find the gateway locations and we create a tree-topology backhaul network. As can be seen, we can benefit a lot by increasing the number of hops, in this map we can connect a $600 \mathrm{~m} \times 600 \mathrm{~m}$ area with 3 gateways and 20 ABSs. Also, the network load is balanced over these GWs such that the maximum load is 7 ABSs per gateway. . . . . . . . . . . . . . . . . . 77 
5.7 Access base stations and gateway densities for different line of sight coverages. We do not cover more than 92 percent, even if we increase the ABS density, as there are some places which do not have line of sight connection with any candidate locations. . . . . . . . . . 78

5.8 Emperical cumulative distribution function of link distances. Data suggest a uniform distribution. Also, the probability of having links longer than $300 \mathrm{~m}$ is less than 20 percent. . . . . . . . . . . . . . . 79 


\section{Nomenclature}

\section{Acronyms}

\begin{tabular}{ll}
\hline Acronym & meaning \\
\hline \hline $5 \mathrm{G}$ & fifth-generation \\
ABS & access base station \\
BILP & binary integer linear programming \\
BS & base station \\
INR & interference-to-noise ratio \\
LOS & line-of-sight \\
LP & linear programming \\
MCP & maximum coverage problem \\
MCPC & maximum coverage problem with connectivity \\
mmWave & millimeter wave \\
MILP & mixed integer linear programming \\
MIMO & multi-input-multi-output \\
NLOS & non-line-of-sight \\
NP & non-polynomial time \\
PPP & Poission point process \\
SNR & signal-to-noise ratio \\
SP & simple polygon \\
SIR & signal-to-interference ratio \\
SINR & signal-to-interference-plus-noise ratio \\
\hline &
\end{tabular}




\section{Chapter 1}

\section{Introduction}

High data rates, coverage and reliability, low latencies, reduced costs, and managing a large number of connected devices are the main targets of fifth generation $(5 \mathrm{G})$ wireless networks. Several technologies are considered as main potential candidates for these requirements. The first technology is to increase the number of base stations (BSs) deployed, referred to as network densification. The second is to increase the received gain using multi-antenna techniques such as beamforming and massive multi-input-multi-output (MIMO). And the third is to increase the bandwidth of the network, which directly translates to an increase in the data rate [1].

Current sub-6 GHz bands are mostly congested and are not able to provide enough bandwidth. Therefore, a shift toward higher carrier frequencies is predicted. The most discussed candidate for $5 \mathrm{G}$ networks is the millimeter wave (mmWave) band, which is nominally the frequencies between $30 \mathrm{GHz}$ and $300 \mathrm{GHz}$ (in practice, usually between 28 and $75 \mathrm{GHz}$ ). The mmWaves can provide hundreds of times more spectrum than the currently allocated bandwidth for broadband mobile communication. However, the mmWave propagation characteristics are qualitatively different compared to those in the lower frequencies. First, mmWaves are much more vulnerable to blockage and do not penetrate common walls and tinted glasses well. Second, the path loss is 
much higher in these frequencies, which limits the communication distance. Several measurement campaigns suggest the possibility of establishing mobile links of around $200 \mathrm{~m}$ in dense urban areas, which means that mmWaves might be a good fit for small cells [2].

Although densifying the network with small cells is a brute-force solution to increase the rate, it is a potential candidate for $5 \mathrm{G}$ networks. Installing a large number of BSs is extremely costly; also, bringing the fiber connection to each individual cell might not be feasible. One enabler technology to reduce the number of wired connected BSs is wireless backhauling; that is, to connect only a small number of the BSs directly to fiber and connect the rest of them via single or multiple-hop wireless backhaul links. These links should be reliable and high capacity in order to forward one entire cell's data to another BS. As access links may use mmWaves, backhaul links need to use mmWaves also to satisfy capacity requirements. As a result, using mmWave line-of-sight (LOS) links is the preferred backhauling scenario [3]. To provide LOS links between BSs we need to plan the network.

Network planning - that is, finding the best BS sites - is an important design step which directly affects the network cost. Also, because of the layout dependency of mmWave networks, due to non-line-of-sight (NLOS) limitations, network planning is more crucial in 5G. Network planning can be divided into two steps [4-7]: the first is to place many good candidate sites and the second is to select a subset of them to provide coverage. The second step usually uses optimization as its main tool. The first step requires the knowledge of the coverage area of each candidate BS site [8]. To find the coverage area, particularly in mmWave networks, we need to model the blockage.

Blockage modeling has been conducted by several researchers using probabilistic models [9-12]. For example, to analyze the network properties, some researchers 
model the buildings as random rectangles in the map, while others model the BS locations with a Poisson point process (PPP). Also, there exist several LOS probability functions used in the literature to model whether or not a link is blocked. However, we believe these models are not detailed enough for our problem. As one of the contributions of this thesis is to calculate several quantitative network properties, such as BS densities, we need to use reliable blockage modeling. Therefore, we use real map data of two large and dense urban areas, Manhattan and Paris, in our study [13].

In this work, we first use computational geometry to place candidates on the walls of the buildings, and then we formulate several optimization problems to select the best sites out of them. We mainly use mixed integer linear optimization to maximize the coverage and minimize the number of the BSs. Also, we formulate greedy algorithms to approximate the optimal solution in a reasonable amount of time.

This thesis's contribution is to plan a small cell network, however, it should be noted that in real scenario networks, the small cell base stations are only a part of a bigger heterogeneous network (HetNet) which consists of macro cells, small cells, and other different kinds of access nodes all working together to bring coverage [14].

Also, the user's movements inside a dense small cell network increase the frequency of handovers; therefore, successful mobility management is critical for future HetNet architecture. Generally, handovers are classified into two major categories: horizontal handovers and vertical handovers. In the horizontal case, handover occurs between two same technology BSs, like the neighboring base stations handover in GSM networks. However, in vertical case, handover occurs between two different technology BSs, For example between a Wi-Fi access point and a cellular base station. For a vertical handover decision, a new radio interface might need to be activated in handheld device as well [15]. Handover handling in HetNet is a discussed issue 
in literature [16] [17]. Vertical handover could be used to be the case to handle the fast moving users by connecting ("camping") them on macrocells, while horizontal handovers could be made by low-speed users.

\subsection{Design Overview}

In this thesis, we estimate the BS densities required for the outdoor LOS coverage with mmWave small cells. Also, we consider wireless backhauling opportunities and estimate the wired connections densities. To approach these questions, we design an automatic network planner to find the BS locations. We have two main concerns in this thesis: first, we want to bring the LOS coverage to a large portion of the outdoor area; second, we want to do this task with as few BSs as possible.

We are studying small cell deployment for 5G networks in this thesis and we assume the mmWave bands are to be used for this kind of networks. Covering 100 percent of the outdoor area is an over engineering approach and in the context of our application we are interested to cover around 90 percent of the outdoor area. We observed that, after 90 percent coverage, the additional coverage area we can have by installing a new BSs is not practically reasonable. To cover the remaining area, we suggest to install some macro/pico cells, using lower frequencies, to compensate the uncovered area in the small cell network.

In network planning, we use the previous works of authors in [13] and place many candidate BSs on the map. The algorithm developed by [13] uses stochastic geometry and finds good local sites for BS places. Then, for a given BS density (K), we maximize the coverage area and sweet the parameter $\mathrm{K}$ to find the minimum BS density required for 90 percent coverage. We formulate our optimization problems as mixed integer linear programming (MILP). 
Following these steps, we consider mmWave LOS wireless backhauling as one of the possible approaches to reduce the number of fiber-connected BSs (gateways) in

the network. As a result, high costs can be avoided by not bringing fiber to every BS in a densified network. Our idea is to place access BSs on the map such that they see each other, i.e., the access BSs create a connected graph, with the LOS backhaul links are the edges of this graph. Then we select a few of these access BSs and connect them to the network via fiber. Our objective is to minimize the wired connections while providing coverage with at most one hop wireless backhaul link.

After planning the BSs, we look through several network properties. The most important ones are the BS densities required for the LOS coverage, wired connection densities, the empirical distribution of the LOS fraction of a cell, and empirical distribution of backhaul link distances. Also, we discuss noise and interference limitations of mmWave planned networks. As we provide simulation results, one important piece of this work is that we use many real maps to ensure statistically representative results.

\subsection{Contributions and Novelty}

Finding the best BS sites, referred to as network planning, has a considerable effect on the network cost. As network planning is interested in best sites, one of its main tools is optimization. Network planning is well studied for lower frequency sub-6 GHz networks. Also, it has been discussed in literature for indoor areas.

However, network planning for next-generation wireless networks should consider different limitations and different opportunities due to changes in the propagation characteristics of higher frequency mmWaves. We believe it has not been investigated in the literature, and is novel. Our contributions can be summarized as follows: 
- The BS density is an important property of any network and is directly transferred to the deployment cost. In this work we find possible BS densities for mmWave networks. The contribution is to find the required density value for covering the user plane with LOS small cells. The density also contributes to many network properties such as interference limitation, latency, and cost.

- Planning a mmWave wireless backhauling network to minimize the number of wired connected BSs. We consider wireless backhaul opportunities and plan the network in order to make small cell deployment feasible. Also, we find the possible gateway densities in the network if we allow single-hop wireless backhaul.

- Approximating the art gallery problem with cooperative guards. In our developed method to plan the wireless backhaul network, we first needed to create a connected graph of BSs to cover the outdoor area. This problem is analogous to the art gallery problem with cooperative guards, where we used the connected subgraph problem to approximate the optimal solution. In an engineering context, we are not interested to cover 100 percent outdoor area, and we cover around 90 percent in our application.

- Using many real maps in our analysis to find more represntative simulation results. Carrier wave propagation is more dependent on the environment due to high penetration loss of mmWaves. Therefore, to find representative results, it is necessary to use real maps. Many building modeling methods do not accurately represent real urban areas. We run the simulations on many maps in two large dense urban cities. 


\subsection{Many Maps}

Because of NLOS limitations of mmWaves, blockage modeling is more important in 5G networks. One can use the probability of LOS function to find out whether a link is LOS or not, like the multi-ball model introduced by [11]. One other option is to use stochastic models to generate building footprints and to place BSs. The authors in [10] placed random-sized and orientation rectangles to model the buildings, which is a good fit in rural areas. To analyze the network properties, authors in [10] and [11] placed BSs according to a PPP. In many situations, these models are the preferred approach, as they can be reproduced easily. However, they might not be accurate enough.

A more accurate approach is to bring real maps into the study. Using real maps first needs to access building footprints data and BS locations. As a result of current advancements on map data and their free availibility, such as the OpenStreetMap project [18], it is possible to access this information and use it as a powerful tool in our study. The authors in [11] used building footprints and real BS sites to validate their multi-ball model. The authors in [19] considered some urban areas in Manhattan and Chicago to gain insights into the coverage and rate performance. However, as we are interested in empirical data, the map area should be large enough for reliability of the simulation results.

Extracting data from small maps may not be representative of what is actually happening in the network. Therefore, to find acceptable data regarding the network properties, one should use many maps to obtain statistically representativ results. In this thesis, we use many maps, first introduced by [13], for dense urban areas. Also, we want to analyze the effect of the city geometry on the network, and we pick the maps of Manhattan Island and central Paris for our analysis. We choose Manhattan because 
it is a newer built city, has straight wide streets and more rectangular buildings. In contrast, streets bend in Paris, buildings are more randomly shaped and the city has

developed over a longer time period. We plan an outdoor network of more than $5 \mathrm{~km}^{2}$ in each city.

\subsection{Organization of the Thesis and Publications}

\subsubsection{Organization}

Chapter 2 is the engineering setup and literature review, which explains the motivations behind this work. In its first section, the need for next-generation wireless networks is explained and some potential enabling technologies are named and briefly explained. Then, we explain the mmWave possible propagation limitations, difficulties and issues. We further discuss the need for planning the network, as well as several opportunities to make such networks feasible. At the end of this chapter, we summarize the ideas presented and state the problem we will solve in this thesis.

Chapter 3 discusses several related research areas, mainly in mathematic literature, which we touch on in this thesis. The sections in this chapter are mostly independent of each other and can be accessed randomly. In this chapter we briefly go through the art gallery problem, the connected subgraph problem, the algebraic connectivity, the visibility polygon, and basic optimization formulations.

Chapter 4 is an expanded version of the first published paper and goes through several ideas regarding the mmWave network planning. In this chapter, we explain the core of our network planner in which we use optimization tools to find the BS sites in real urban areas for the outdoor LOS coverage. This chapter includes the explanation of the maximum coverage problem, and also includes our contribution regarding the possible BS densities in mmWave wireless networks. 
Chapter 5 is an expanded version of the second submitted paper. It uses the wireless backhaul opportunity, an important technology to reduce the high density of the wired connected BSs, and tries to find the best sites for fiber connection. This chapter includes the explanation of the connected subgraph problem and the set coverage problem. This chapter also shows the combined benefit of wireless backhauling and network planning as a tool to effectively reduce the number of BSs.

\subsubsection{Publications}

1. Nima Palizban, Sebastian Szyszkowicz, and Halim Yanikomeroglu, "Automation of millimeter wave network planning for outdoor coverage in dense urban areas using wall-mounted base stations", IEEE Wireless Communications Letters, vol. 6, no. 2, pp. 206-209, April 2017.

2. Nima Palizban, Sebastian Szyszkowicz, and Halim Yanikomeroglu, "Millimeter wave line-of-sight access and backhaul network planning with wall-mounted base stations", under review in IEEE Communications Letters (submission: 28 July 2017). 


\section{Chapter 2}

\section{Literature Review and Problem Statement}

\subsection{Next Generation Wireless Networks Overview}

Since the telecommunication standardization began, each generation was a paradigm shift to satisfy new emerging applications and importantly, not just an incremental version of its previous one [1]. For instance, the 2G networks, mainly GSM, aimed to provide voice communication to its users. A paradigm shift occurred when $3 \mathrm{G}$ standards, such as HSPA, HSPA+, and WiMax, came into existence with data communication, the internet, and multimedia as their main targets. Continuing through the path, LTE aimed to provide video transmission, where video was becoming main load of the traffic. It is predicted that video traffic will be 82 percent of all consumer Internet traffic by the year 2020 [20]. Next-generation wireless networks i.e., 5G is mainly driven by the explosion in data traffic due to high-definition video transmission, emerging applications such as virtual reality, and possibly many other unforeseen applications.

Furthermore, compared to previous generations, 5G aims to support a wide variety of applications with different requirements. $5 \mathrm{G}$ is predicted to handle high data rate applications, such as live video presence and mobile video for cars. Also, it is desired 
to support low latency and high reliability required applications including, possibly, driverless cars and public safety. Another important category of $5 \mathrm{G}$ applications is the internet of things (IOT); as a result, the number of connected devices will potentially be higher than today's networks. Handling this diverse range of applications is a challenge for $5 \mathrm{G}$.

Several different technologies might be used for future networks, mainly to provide high required data rates. All these technologies' main ideas are based on the following fundamental rate equation:

$$
\left.R=n W \log _{2}(1+S N R), \quad \text { [bits per second }\right]
$$

where $W$ is the bandwidth of the link, $S N R$ is the signal-to-noise ratio, and $n$ is the MIMO gain. The main contributing technologies are categorized as follows:

- Densifying the network,

- Increasing the bandwidth associated with each cell,

- Using multi-antenna technologies.

One possible way to increase the rate is to reuse the bandwidth by deploying more infrastructure, in order to densify the wireless network. Although it is a brute-force way to increase the rate, it is one of the possible themes for next-generation wireless networks [1]. The heterogeneity of different classes of base stations (e.g., macro cells and small cells) provides flexible coverage areas and improves spectral efficiency. Also, reducing the size of the cell increases the spectral efficiency, due to lower path loss, which is a favourable side effect of densification [21].

Increasing the bandwidth directly increases the rate, as can be seen in (2.1). Currently utilized congested bands do not allow the bandwidth increase, therefore 
pushing the $5 \mathrm{G}$ networks to move forward to higher frequency bands where there is a lot of bandwidth available [22]. There is an order of magnitude more bandwidth in the mmWave band, usually referred to as frequencies between $30 \mathrm{GHz}$ to $300 \mathrm{GHz}$, and these bands are considered as the main candidates for 5G networks [19]. Moreover, advances in device technologies to allow them to work at mmWave frequencies are enabler steps for $5 \mathrm{G}$ networks [23].

Another important enabler technology is to increase the spectral efficiency by beamforming, that is to increase the directivity of the antennas, mainly on the base station, to increase the received power [24]. As the frequency increases, the wavelength decreases and, as a result, practical antennas can be designed in smaller dimensions (as a rule of thump, $\lambda / 2$ represents the possible antenna size), which brings the possibility of packing larger number of antennas into the same physical area. Therefore, multi-antennas technologies such as MIMO are anticipated to play a key role in 5G networks [25]. Massive MIMO, which involves packing thousands of antennas on transceiver devices, can also be considered as a potential technology.

\subsection{Millimeter Wave Propagation}

As explained in Section 2.1, mmWaves are one of the candidate bands to be used in $5 \mathrm{G}$ networks. However, mmWaves have qualitatively different propagation characteristics than current sub-6 $\mathrm{GHz}$ bands. Most noticeably, mmWaves are sensitive to physical blockage and, secondly, the path loss is much higher in these frequencies. According to the Friss equation, the free space path loss increases at the rate of $20 \mathrm{~dB}$ per decade by increasing the frequency, which is due to the smaller antenna effective area in higher frequencies. The Friss equation is as follows: 


$$
P_{r}[d B]=P_{t}+G_{t}+G_{r}-P_{L},
$$

where $P_{r}$ and $P_{t}$ are the recieved power and transmitted power, respectively, and $G_{r}$ and $G_{t}$ are the received and tranmitted antenna gains. $P_{L}$ is the path loss, which is stated as follows:

$$
P_{L}(d)[d B]=P_{L}\left(d_{0}\right)+10 n \log _{10}(d)+20 \log _{10}(f),
$$

where $P_{L}\left(d_{0}\right)$ is the path loss at the reference point, $d$ is the distance in meters, and $f$ is the frequency. The high path loss in millimeter bands does not allow on to have large cells. $200 \mathrm{~m}$ cell radius is discussed in literature as a possible communication range [2], [26] and [22]. We assume this distance to be feasible for LOS communication.

The other main characteristic is that mmWaves are much more vulnerable to physical blockage. There are several measurement campaigns done by [2] in which they studied $28 \mathrm{GHz}$ and $73 \mathrm{GHz}$ waves, noting that the penetration loss from a typical office wall can be as high as $40 \mathrm{~dB}$. Penetration loss is much higher at mmWave frequencies, and future buildings are also expected to use different materials for energy considerations, such as tinted glasses which isolate the indoor and outdoor areas [27]. As a result, the indoor and outdoor areas are likely to be more isolated from each other and each needed to be covered with different cells [28].

There is also a concern about the reliability of the mmWaves links, as they are affected by rain and snow. However, when one considers the fact that today's cell sizes in urban environments are on the order of $200 \mathrm{~m}$, it becomes clear that mmWave cells can overcome these issues. Particularly at $28 \mathrm{GHz}$, only $7 \mathrm{~dB}$ per $\mathrm{km}$ of attenuation is expected due to heavy rainfall, which translates to only $1.4 \mathrm{~dB}$ attenuation over 
$200 \mathrm{~m}[2]$.

Building dimensions are much larger than the wavelength in these frequencies, which makes the waves harder to diffract from the corners and not to propagate from one street to another. As diffraction is lower and penetration is high, the LOS communication differs sharply from NLOS communication; and NLOS scenario is not preferred due to low power received in most areas. To summarize, the main propagation differences between mmWaves and sub-6 GHz waves are as follows:

- There is a sharp difference between LOS and NLOS links,

- Because diffraction is low, the NLOS condition is mainly due to reflection and scattering,

- Indoor to outdoor penetration loss is noticeably high for many common materials, and it is usually not possible for the outdoor BS to cover the indoor area,

where all these differences should be counted in an appropriate channel model.

\subsection{Wireless Backhauling}

To satisfy the required rates in 5G networks, one of the key enabler technologies is to increase the density of the BSs by installing many small cells in the outdoor area [29]. The small cell deployment is also encouraged by the fact that mmWave communication might not be feasible for large distances due to high path loss, and the possible distance is around $200 \mathrm{~m}$ for access links. However, deploying the high number of BSs is costly. Bringing wired connection to all the BSs as well as the high number of infrastructures is an important prohibitive factor in installing the small cells [30]. To reduce the number of wired connections, and to reduce the high cost 


\begin{tabular}{|c|c|}
\hline Backhauling option & Advantages \\
\hline LOS links & higher capacity \\
NLOS links & does not need alignment, lower deployment cost \\
Microwave frequencies & longer link distances, less vulnerability to blockage \\
mmWave frequencies & more bandwidth available, smaller antenna sizes \\
\hline
\end{tabular}

Table 2.1: Brief comparision of different wireless backhaul options.

of bringing fiber to each BS, one can use the wireless backhauling opportunity to connect the BSs via wireless links and connect only a small subset of BSs via fiber to the network. That is to say, wireless backhauling can bring cost feasibility to future networks.

There are several different options when it comes to wireless backhauling, including LOS or NLOS backhauling, and using microwave or mmWave carrier frequencies. In urban settings, small cell backhaul links are more likely to be blocked by multiple obstructions from longer distances [31], emphasizing the need to communicate in NLOS scenarios. Some papers suggest microwave band as a potential candidate for NLOS backhauling [32]. Microwaves might be used more than mmWaves in longer distance links and may have better performance in NLOS links [33]. Table 2.1 summarizes several advantages of the stated wireless backhauling options.

The backhaul link should have enough capacity so that it can forward a large amount of data collected from all of its assigned users. To provide the enough data rate and capacity, one solution is to increase the bandwidth assigned to the link. There is plenty of bandwidth available at mmWave frequencies, especially the $73 \mathrm{GHz}$ band. If the access links are already using mmWaves, choosing mmWave backhauling would be more realistic as it can bring enough capacity [3]. Also, the feasibility of mmWave transmission within a range of a few hundred meters has recently been 
proven by systematic outdoor measurements [34]. We are interested in the mmWave LOS backhauling option and we plan the network such that BSs see each other. Wireless backhauling is the main idea of Chapter 5 .

\subsection{Network Planning}

Placing BSs in proper sites is highly important and directly affects the network cost. In this work, we refer to the BS placement problem as network planning, as it is actually a major part of it. Network planning is generally considered as a combinational optimization problem, where the planner selects a subset of possible BS sites to cover the entire area, or a high percentage of the desired area, with the minimum number of installed BSs. Network planning is usually separated into two independent branches: indoor network planning and outdoor network planning. One possible approach is to divide the planning into two steps. First is to place many candidates in the area and find their coverage. Second is to select a subset of candidates to jointly optimize the coverage requirements.

Finding the BS places is one of the topics studied in literature, mostly for sub-6 GHz bands. The authors in [4] and [8] used genetic algorithms for selecting the possible BS sites for radio frequency waves. They first find each candidate site's coverage area by a radio wave propagation simulation piece of software called ParFlow ++ ; to find the best sites, they tried to solve the problem with a bio-inspired genetic algorithm. Genetic algorithms generally do not give an optimal solution.

In addition, there are several studies on indoor network planning. One such study uses integer linear programming in interference limited networks to find the best BS sites [5] for CDMA systems. Also, the authors in [35] tried to approach the BS placement problem by means of swarm intelligence. And the authors in [6] used 
a hybrid algorithm to consider the signal-to-interference ratios in both forward and reverse links.

Network planning for the mmWave outdoor area is not well-studied and it is fairly new in the literature, we consider [13] as one of the first papers to touch on this topic. The network properties are fairly different with the sub- $6 \mathrm{GHz}$ waves, for example, the interference limitation assumption in current networks may not apply to mmWaves to the same extent. In general, the following two differences should be considered: first, mmWave propagation is more dependent than lower frequencies on LOS and the exact placement of a BS strongly affects its LOS coverage. Second, city geometry and street shapes affect the network more than lower frequencies. These two major reasons magnify the importance of network planning for $5 \mathrm{G}$.

\subsection{Blockage Modelling and Real Maps}

The environment affects many network properties; particularly in 5G networks with possible millimeter wave signals, this environmental influence is increased. The sharp difference between NLOS and LOS channel models is a result of blockage and it is correlated to the city geometry. In general, blockage modeling is one of the necessary steps to analyze the millimeter wave networks. In this section, we describe some of the methods described in liturature to model the blockage.

The authors in [10] and [9] used the Poisson point process (PPP) model to distribute the BSs on the map. Then, to consider the blockage effect, they modeled the buildings of random location, size, and shape with a random process of rectangles, called boolean scheme in shape theory. In a Boolean scheme model, the centers of the rectangles are distributed according to a PPP, and their orientations and sizes are 
identically and independently distributed (iid) uniform random variables. By modeling the BS places and building locations, the authors analyzed the possible rates and coverages in millimeter wave networks.

Instead of working with empirical data and finding the LOS or NLOS links in its brute force, the authors in [11] introduced a muti-ball blockage model along with a PPP BS places model to find whether a link is operating in the LOS or NLOS scenario. In the multi-ball model, they used a piece-wise function of the distance between the user and the BS to approximate the probability of having a LOS link. That is, to split the BS-to-user distance in several regions, and in each region, the probability of haveing a LOS access is the same. The authors also tried to validate their model using real BS sites and building footprints in a metropolitan area in London, UK, obtaining their BS data from OFCOM [36] and building data from OS [37].

In this thesis, as we wanted to find BS densities, our main contribution, we needed to move toward real maps to increase the accuracy of our results. We obtained our building footprints data from OpenStreetMap project [38], which is an open source database. OpenStreetMap has the potential to engage volunteers from around the world, including less developed regions, and it is updated regularly [18]. According to the authors in [39], the OpenStreetMap database has a high positional accuracy and may be used for small and medium scale mapping applications. Furthermore, the areas of high accuracy are primarily located in the highly populated urban centers, which make it a proper fit for our study.

Fig. 2.1 shows four different map categories, where important differences can be seen between them. The first difference between two areas can be readily stated as the difference between the density of the buildings (the fraction of total area filled) in the map. The second one is the difference between the shape of the streets and buildings in an area. We found that narrow bending streets increases the BS density 
required for a network, as the street shapes limit the range of LOS coverage of a BS.

\subsection{Proposed System Design}

Small cells are a potential candidate for future networks or even current networks. It is also a good fit for mmWaves, as the cell radius is fairly small (approximately $200 \mathrm{~m}$ according to [2]) due to the high path loss in higher frequencies. However, deploying a large number of BSs would be costly, both because of the BS cost and installing the fiber connection to the BS. In this thesis we have tried to plan the network - that is, to find the possible sites for BSs - in a way to minimize both the number of BSs and fiber connections.

We limit the problem to planning the mmWave networks with small cells, as this is predicted to be the theme in $5 \mathrm{G}$. Also, we only consider the LOS communication and we treat the areas with no LOS access to any BS as an outage. We use wallmounted BSs, which are BSs placed on the walls of the buildings. We do not take the height of the BSs into account; however, higher BSs are preferred because of the obstacle blockages in the streets [40]. We consider outdoor area only, as the indoor and outdoor areas are isolated from each other, and indoor network planning is a separate research area. Then, to model the coverage, we distribute points on a dense square grid in the outdoor area.

Wall-mounted BSs are probably going to be small and can be easily installed on the buildings. There is an ongoing discussion on where to put the BSs, with some options being lamp post, above rooftops, traffic lights, etc. We picked the wallmounted option mainly because of the fact that the data of the walls are available in open source maps, whereas, lamp post, traffic lights, and other similar objects' data is not as readily available. Also, above-rooftop BSs might have the LOS problem, as 



Figure 2.1: Different maps, Manhattan map on the top left, Paris on the top right, with two toy maps, Manhattan grid on the bottom left and random rectangles on the bottom right. It can be seen that different cities have different outdoor area shapes which affect the line-of-sight area from a base station. Bending streets in Paris and straight long streets in Manhattan affect the networks in different ways. Also, a random rectangle map might not be similar enough to dense urban areas, and would be a better fit for rural areas. 




Figure 2.2: Engineering setup. As LOS communication is preferred, mmWave backhauling can bring connection to areas not visible by the gateway.

their coverage might simply be blocked by the roof of the building they are mounted on.

To reduce the cost spent on bringing fiber connection to many access BSs (ABSs) in the network, we consider wireless backhaul between the BSs. We plan the network so that BSs see each other via LOS links. Fig 2.2 shows the basic concepts, such as wall-mounted BSs, and other engineering setups we assumed.

The network planning is divided into two main steps: first we place many candidates on the map, and then we select a subset of the candidates to bring the LOS coverage. Candidate placement is done with computational geometry tools and selecting the best BS sites is done with optimization tools. In Chapter 4 we only consider 
the coverage problem, and in Chapter 5 we use wireless backhaul opportunities to further decrease the number of wired connections. 


\section{Chapter 3}

\section{Related Topics}

In this chapter, we discuss several different topics which are related to our problem and share some similar ideas. The sections in this chapter are designed to be independent from each other. The material is mainly theorems already presented in the mathematics literature which are not stated much in the literature on wireless communications. Also, we try briefly discuss several ideas we go through in this work which leads us to our final solution in Chapters 4 and 5, but are not directly included in our formulations.

It should be noted that some of the materials in this chapter are not directly used in our solution. Particularly, Section 3.2 to introduce some of the current existing studies in the art-gallery problem literature, but is not used in our approach to solving the problem. We are interested in an engineering application and in this context, for example, covering 100 percent of the outdoor area is not desired and we are mainly interested to cover a large portion (around 90 percent) of the desired area. We

develop our own method to approximate the art gallery problem which is introduced in Section 3.4. 


\subsection{Visibility Polygon}

Higher carrier frequencies are more sensitive to blockage, do not penetrate well, and do not usually diffract. As a result, the preferred propagation scenario is LOS. Also, as described in Section 2.6, we consider NLOS areas as outage. In order to find the covered area, calculating the visibility polygon of each BS is crucial.

In computational geometry, the visibility polygon or visibility region for a point $P$ in the plane among obstacles is the polygonal region of all points of the plane visible from $P$. There are several algorithms available for its evaluation in [41] and [42]. One can use the open source software VisiLibity [43] to find the visibility polygon in simulations.

In addition to the visibility polygon, we need to consider the cell radius to find the practical coverage area of a BS. We assumed a $200 \mathrm{~m}$ cell radius, so the coverage area is simply the visibility polygon intersected by a cricle of radius $200 \mathrm{~m}$. Fig 3.1 shows the resulting visibility polygon of a random point in the outdoor area.

\subsection{Art Gallery Problem}

Our problem, as stated in previous chapters, is to find the best BS sites for the outdoor coverage. The next-generation wireless networks are possibly going to use higher carrier frequencies for data communications, particularly mmWaves. In our problem statement, we considered mmWave communication to be practical for the LOS regions only. Actually, modeling the problem this way is analogous to a version of a problem named the art gallery problem in mathematics literature. It is a wellstudied visibility problem in computational geometry, which is introduced in the next paragraph. 


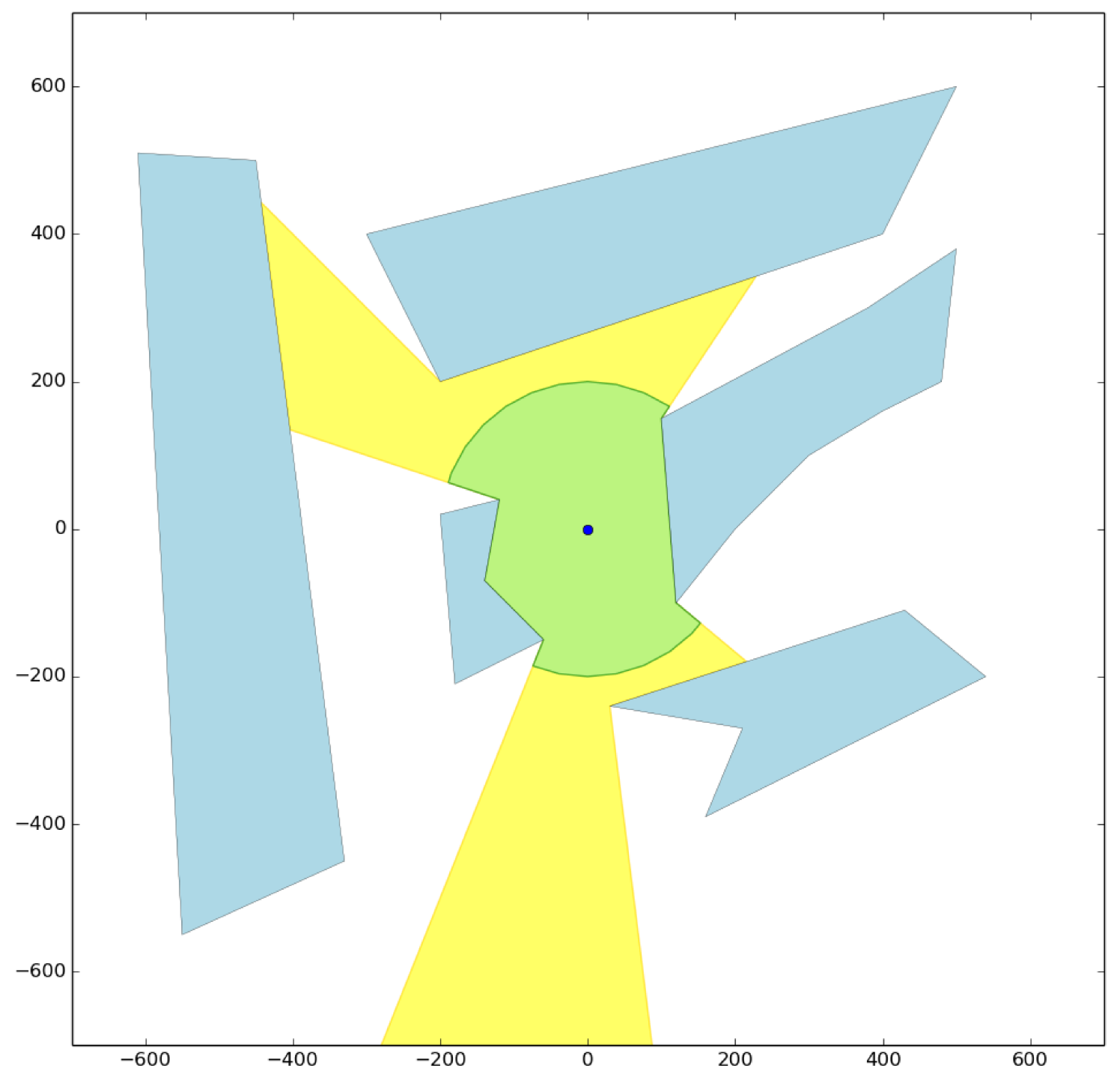

Figure 3.1: Visibility Polygon, the center point is represented with blue, the yellow area is the visibility polygon associated to the center, and the green area is the coverage within $200 \mathrm{~m}$ cell radius. 


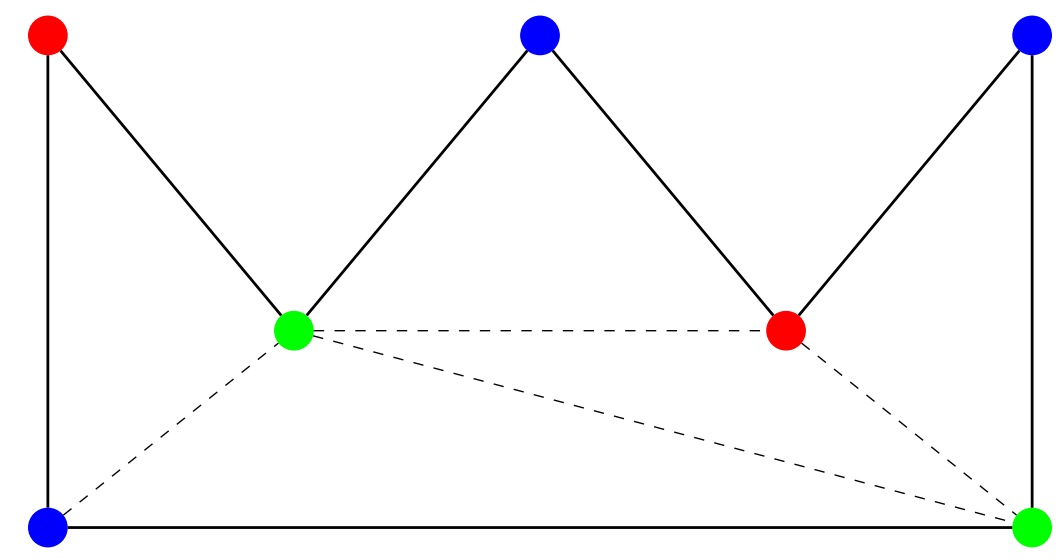

Figure 3.2: Upper bound on the number guards to cover a simple polygon art gallery. A coloring argument can be used to show that it is possible to guard the gallery with $\lfloor n / 3\rfloor$ guards, where $n$ is the number of vertices. Here, if we pick the green color, then 2 guards are enough which is equal to $\lfloor 7 / 3\rfloor$. The dashed lines show the triangulation of the gallery.

A polygon $P$ is an ordered sequence of points $p_{1}, \ldots, p_{n}, n \geq 3$, called the vertices of $P$ together with the set of line segments joining $p_{i}$ to $p_{i+1}, i=1, \ldots, n 1$ and $p_{n} t o p_{1}$, called the edges of $P$. $P$ is called simple if any two non-consecutive edges do not intersect [44]. Now consider that we have a simple polygon referred as the art gallery. The problem is to find a collection of points, referred as guards, to fully guard the art gallery. A point in the art gallery is guarded if it is inside the visibility polygon of at least one guard.

The art gallery problem was first named by Vaclav Chvatal [45], when he gave the upper bound on the number of guards needed to guard the gallery. By using triangulation it is simple to prove that $\lfloor n / 3\rfloor$ guards are always sufficient and sometimes necessary to guard a simple polygon with $n$ vertices. A triangulation of a polygon is the decomposition of the polygon into triangles using non-crossing diagonals. Each triangle is guarded by all of its vertices, assuming we use three different colors and 
assign each vertice a color such that two vertices at the end of the same edge never have the same color. Clearly, each triangle has all three colors, if we choose one of the colors and place a guard at all locations with the same color, then the gallery is covered. Since no color is repeated in a triangle we need at most $n / 3$ guards. Fig. 3.2 shows the concept.

\subsubsection{Art Gallery Problem with Cooperative Guards}

The art gallery problem has many different variations. In Section 4 we are mainly interested in solving the art gallery problem with holes (each building is a hole in the outdoor coverage area). Also, in Chapter 5, to bring backhaul opportunities, we are interested in planning a network such that the BSs see each other, and we want the BSs to create a connected graph. This problem is referred to in the literature as the art gallery problem with cooperative guards [46]. That is, to minimize the total number of guards such that each guard can transfer its information to any other guard, at least from one path. The name cooperation comes from the idea that if a guard is in danger it can communicate with others and cooperate with them to face the problem. There are two variations of this problem, namely weekly cooperative and strongly cooperative guards, where in the first variation each guard is required to be seen with at least one other guard (guarded guards) [47]. In the strongly connected guards, however, the entire graph of the guards should create a connected graph and there should be at least one path between any pair of guards. It is shown by induction that the minimum number of connected guards which are necessary to watch any polygon with $n$ sides is $\lfloor(n-2) / 2\rfloor$. This result was originally established by Hernandez Penalver [48]. 


\subsubsection{Art Gallery Problem in Practice}

In the engineering context, covering 100 percent area is not necessarily desired. In almost all the cases if one covers a high percentage of the area one can satisfy the coverage requirement. In this thesis, we are not interested in covering the entire area and we aim for 90 percent coverage. We observed in our results that if we want to achieve more than 90 percent coverage, then we need to dramatically increase the number of guards, that is to say, the average area covered by each guard decreases and it might not have an economic justification.

Furthermore, we work with real building footprints in our problem, and buildings can have many different shapes by their nature. They can have a large number of vertices in their corners only. They might have some narrow open areas inside them, like small backyards, which do not have easy access to the outdoor area and need a BS devoted to itself. These difficulties make the developed approaches in mathematics literature (for 100 percent coverage) unstable in an engineering point of view.

Our approach to the art gallery problem is different. Instead of using coloring algorithms, we use optimization tools and model the problem as a set coverage problem, similar to the authors' approach in [49]. That is, we distribute points in the outdoor area and we aim to maximize the total number of points covered. Also, we are interested in the number of guards needed to cover around 90 percent of the outdoor area.

\subsection{Graph Connectivity}

In our problem statement, particularly in the backhaul network planning, we have several points in our maps as candidate locations, access BSs, and gateways, which are desired to be connected to each other through the LOS links. These concepts suggest 
the use of graphs in the analysis, that is to consider the BSs as the graph nodes and the wireless links as the graph edges. Studying graphs will help us to analyze several network properties. To start with, we will give some basic graph terminologies in the next paragraphs. These terminologies are later used in Chapters 4 and 5.

Assume we have a graph $G(V, E)$, where $V$ is the set of all nodes (vertices) and $E$ is the set of all edges. In our problem, we have the graph of candidate locations, called $G_{0}$, and we want to select a few candidate locations to create the graph of access BSs, called $G_{1}$. That technically means $G_{1}$ is a subgraph of $G_{0}$. A subgraph is defined as follows: let $G_{0}$ be a graph with vertex set $V\left(G_{0}\right)$ and edge set $E\left(G_{0}\right)$, and similarly let $G_{1}$ be a graph with vertex set $V\left(G_{1}\right)$ and edge set $E\left(G_{1}\right)$. Then, we say that $G_{1}$ is a subgraph of $G_{0}$ if $V\left(G_{1}\right) \subseteq V\left(G_{0}\right)$ and $E\left(G_{1}\right) \subseteq E\left(G_{0}\right)$ [50].

In Chapter 5 we are mainly interested in connected graphs. To define connectivity clearly, we first need to define walks and paths. A walk is simply a sequence of alternating vertices and edges of a graph (starting with a vertex), such that $e_{i}$ in the sequence has ends at $v_{i}$ and $v_{i+1}$. A walk can be written as follows:

$$
W=v_{0}, e_{0}, v_{1}, e_{1}, \ldots, v_{k}, e_{k} .
$$

A path is simply a walk with distinct vertices [51]. Now that we have defined the path, a connected graph can be readily defined as a graph where there is at least one path between any pair of its vertices. There are two other kinds of graph connectivity definitions which might be useful for future work in this area, referred to as the edge connectivity and the vertex connectivity of a graph. Edge connectivity is defined as the minimum number of edges which should be removed from a graph until it becomes disconnected. Vertex connectivity is defined as the minimum number of vertices which should be removed from a graph until it becomes disconnected. These parameters of 
a graph can be used to measure the density of connectivity. It might be interesting to use them in network planning. For example, if the reliability of the network is critical, then we can increase the edge connectivity of $G_{1}$, such that there are more backhauling opportunities for the network.

In Chapter 5, we are looking for a set of conditions to ensure connectivity. The first thing that came to mind was to use the graphs' algebraic connectivity. To cover this material, we first need to define the adjacency, degree, and Laplacian matrixes of a graph. For a given graph $G(V, E)$ with $n$ nodes, we create the adjacency, $A=\left\{a_{i j}\right\}_{n \times n}$, such that $a_{i j}$ equals 1 if and only if there is an edge between node $i$ and node $j$. Otherwise $a_{i j}$ equals 0 (note that the main diagonal is 0 ). The degree matrix, $D=\left\{d_{i j}\right\}_{n \times n}$, is a diagonal matrix with its elements $d_{i} i$ equals to the degree (number of edges going through) of node $i$. Defining $D$ and $A$, the Laplacian matrix is defined as follows: $L=D-A$. An example graph is given in Fig. 3.3, with its corresponding matrices as follows:

$$
D=\left(\begin{array}{lllll}
1 & 0 & 0 & 0 & 0 \\
0 & 1 & 0 & 0 & 0 \\
0 & 0 & 3 & 0 & 0 \\
0 & 0 & 0 & 2 & 0 \\
0 & 0 & 0 & 0 & 1
\end{array}\right), \quad A=\left(\begin{array}{ccccc}
0 & 0 & 1 & 0 & 0 \\
0 & 0 & 1 & 0 & 0 \\
1 & 1 & 0 & 1 & 0 \\
0 & 0 & 1 & 0 & 1 \\
0 & 0 & 0 & 1 & 0
\end{array}\right), \quad L=\left(\begin{array}{ccccc}
1 & 0 & -1 & 0 & 0 \\
0 & 1 & -1 & 0 & 0 \\
-1 & -1 & 3 & -1 & 0 \\
0 & 0 & -1 & 2 & -1 \\
0 & 0 & 0 & -1 & 1
\end{array}\right) .
$$

The Laplacian matrix is used to find whether or not the graph is connected. Assume that $L(G)$ has $n$ eigenvalues as follows:

$$
\lambda_{1}=0 \leq \lambda_{2}=a(G) \leq \lambda_{3} \leq \ldots \leq \lambda_{n},
$$




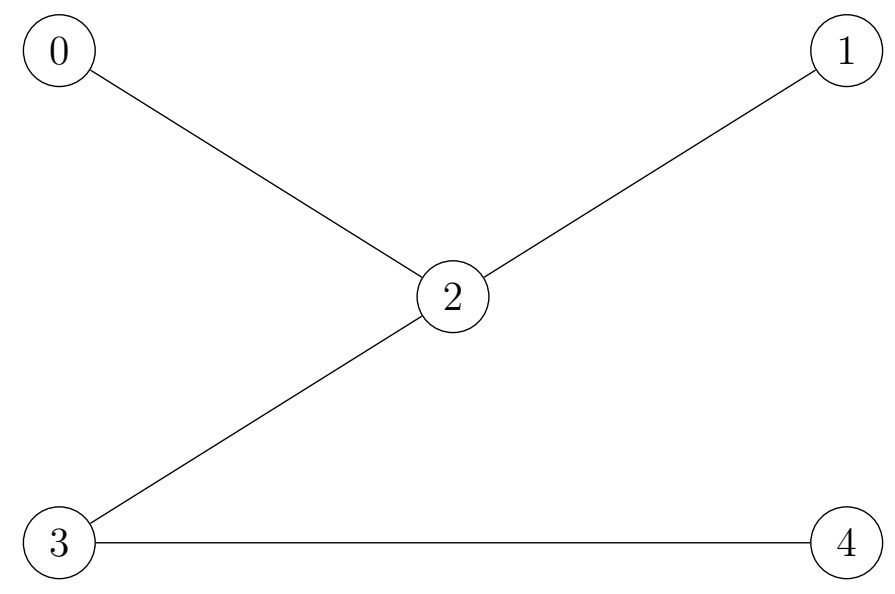

Figure 3.3: A five node connected graph.

the second smallest eigenvalue of the Laplacian matrix, is named the algebraic connectivity of a graph, $a(G)$, and it has been shown by Fiedler that if the algebraic connectivity is positive then the graph is connected [52].

Using algebraic connectivity looks promising at the first sight since we want to check the connectivity of the graph of access BS. If we could somehow find the second smallest eigenvalue of the resulting graph, we would be able to solve our problem, which is:

1. Maximize the coverage,

2. Subject to: $a\left(G_{1}\right)>0$.

However, computing the eigenvalues of a graph is not a linear operation in general. Therefore, as we are looking for a linear optimization problem, this approach is not very helpful for this problem. For example, to find the algebraic connectivity of the 
graph in Fig. 3.3, we should solve the following algebraic equation:

$$
\operatorname{det}(L-\lambda I)=0, \quad \Rightarrow-\lambda^{5}+8 \lambda^{4}-20 \lambda^{3}+18 \lambda^{2}-5 \lambda=0 .
$$

As part of future work, one might try to find the repetition of the zero in the equation. If it is repeated only once, then the graph is connected.

We use a totally different approach for formulating the problem by using network flow concepts, which is described in Section 3.4 and Chapter 5. As a future work, however, finding a linear lower bound, on the algebraic connectivity might be helpful, as it can decrease the high number of added variables for network flow optimization. There are some useful papers regarding the lower bounds of the Laplacian graph eigenvalues, such as [53] and [54].

\subsection{Connected Subgraph Problem}

Finding the algebraic connectivity of a Laplacian matrix is not an easy way to approach this problem, as described in the previous section. Here we look at the problem as a network flow and use a problem already studied in mathematics literature, namely the connected subgraph problem, to solve our problem. The method introduced in this section creates the main idea of Chapter 5 .

As stated in Section 2.6, our aim is to reduce the BSs density for the outdoor LOS coverage. Also, we consider wireless backhauling as a key enabler to deploy small cells, where it can significantly reduce the number of fiber-connected BSs on the map. As a result, we are mainly interested in covering the area with BSs which see each other.

We distribute many candidate locations on the map and create the candidate locations graph, called $G_{0}$. Then we aim to select a connected subgraph of $G_{0}$ to 
maximize the coverage. This problem is some times referred to as maximum-weight connected subgraph problem [55].

We are looking for a set of linear constraints that, if satisfied, make sure the subgraph's connectivity. Assume we want to find a $K$-subgraph, then the main idea is as follows: first, we force each selected node to inject 1 unit of flow into the network, which is to consider the selected nodes as source nodes. Further, we force the flow to only move from one to another if an edge exist between two end nodes (the nodes see each other). Then, if only one sink node exist which expels $K$ units of flow from the network, the subgraph becomes connected.

To model the problem as a network flow, we add a binary phantom link $b_{i}$, entering node $i$, where $b_{i}$ is 1 if and only if the node $i$ is selected. The binary variable $B=$ $\left\{b_{i}\right\}_{1 \times n}$ represents the source node's selection. Also, we add another phantom link $S=\left\{s_{i}\right\}_{1 \times n}$ to each node, such that $s_{i}$ is one if the node $i$ is the graph's sink node. A flow can go from one node to another if the two ending nodes see each other (i.e., $a_{i j}$ is 1 in the adjacency matrix 3.3). Also, if any of the nodes $i$ or $j$ is not selected $\left(b_{i}\right.$ or $b_{j}$ is 0$)$, no flow can go between these nodes, resulting in constraints 3.4.4. To prevent negative flow in the links, we create a directional graph with link variables $X=\left\{x_{i j}\right\}_{n \times n}$ such that $x_{i j}$ represents the amount of flow from node $i$ to node $j$. The idea is summarized in Fig. 3.4.

Finally, if we want to select a connected subgraph with $K$ nodes from a graph of 


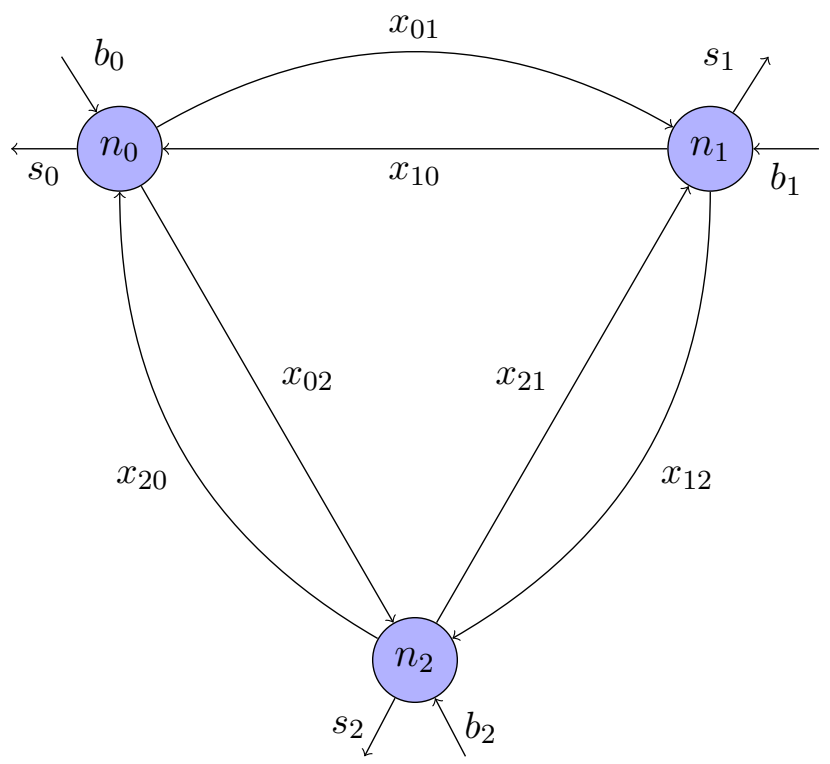

Figure 3.4: A network flow diagram. The flow conservation assumtion for node 1 implies $b_{1}+\left(x_{01}+x_{21}\right)-\left(x_{12}+x_{10}\right)=s_{1}$.

$n$ nodes, the set of linear constraints to ensure connectivity becomes as following:

$$
\begin{array}{lr}
\sum_{i=1}^{n} b_{i}=K, & \\
\sum_{i=1}^{n} s_{i}=1, & \\
\sum_{j=0}^{n}\left(x_{j i}-x_{i j}\right)+b_{i}=K s_{i}, & i \in\{1, \ldots, n\}, \\
x_{i j} \leq K b_{i}, K b_{j}, K a_{i j}, & i, j \in\{1, \ldots, n\}, \\
b_{i} \in\{0,1\}, \quad s_{i} \in\{0,1\}, & i \in\{1, \ldots, n\}, \\
0 \leq x_{i j} \leq K, & i, j \in\{1, \ldots, n\},
\end{array}
$$

where the constraint 3.4.3 is the flow conservation assumption, and 3.4.6 comes from the fact that there are no more than $K$ units of flow in the network in total. This set of constraints can be used in different network optimization problems, and we later 
use these conditions to provide connectivity between access BSs in the network.

\subsection{Integer Linear Programming Terminology}

In this thesis we make extensive use of optimization. It is one of the main building blocks of this work. Although our optimization is not going to give us the optimal solution to the main general problem, it finds an acceptable solution. The founded solution is not optimal because the problem statement to some extend depends on the results of previous steps, and the optimization is only one part of the approach. Therefore in general, the resulting solutions are not the best-planned network. Our problem is to smartly select a number of candidate locations to maximize the coverage, but we are not taking into account many other non-candidate locations which might jointly give us better results. One can enhance the solution by placing more and more candidates on the map. However, placing more candidates quickly increases the complexity of the optimization problem. The other influential parameter is the shape

of each map, there is always the possibility of misinterpreted data in the maps which can directly affect the result of the network planner. However, given the same inputs to the optimization formulations, we find the optimal solution and, in this sense, it is the best.

The optimization formulation is generally stated as follows:

$$
\begin{array}{cl}
\underset{X}{\operatorname{Maximize}} & f(X), \\
\text { subject to } & f_{i}(X) \leq b_{i}, \\
& L \leq X \leq U,
\end{array}
$$


where $X=\left\{x_{i}\right\}_{1 \times n}$ is the optimization variable, $f(X)$ is the objective function, $L=\left\{l_{i}\right\}_{1 \times n}$ and $U=\left\{u_{i}\right\}_{1 \times n}$ are the lower and upper bounds on the variables, respectively, and $i$ functions in 3.5.2 are constraints. Any solution that satisfies all the constraints is called feasible and the set of all feasible solutions is referred to as the feasible set. The optimal solution is the one which gives the best (maximized or minimized) objective function amoung all solutions in the feasible set [56].

In this thesis, we just use linear programming (LP) - that is, when we have the objective function and all the constraints as linear functions of the optimization variables. LP can be written in its general form as follows:

$$
\begin{aligned}
\underset{X}{\operatorname{Maximize}} & \sum_{i=0}^{N-1} c_{i} x_{i}, \\
\text { subject to } & \sum_{i=0}^{N-1} a_{i j} x_{i} \leq b_{j}, \quad j \in\{0,1, \ldots, M-1\}, \\
& L \leq X \leq U,
\end{aligned}
$$

where the optimization variables can be continuous or discrete (in the case of discrete variables, the optimization formulation is referred to as the mixed integer LP) [57]. The mixed integer LP (MILP) is known as non-polynomial time (NP) problem, but non-integer LP problems can be solved in polynomial time, which places them in $\mathrm{P}$ class [58].

Our approach to solving the optimization problems is first to introduce a greedy method and approximate the solution. Greedy methods are heuristics and might not give us the optimal solution. Second, we explain the actual optimization formulation, 
which is mostly a MILP. And finally, we relax the integer constraints to solve a less complex $P$ problem and to found a bound on our simulation results. These bounds are useful when finding the optimal solution is hard and takes too much time, because it can be a strong performance analyzer of the greedy methods.

To solve the optimization formulations, we use different solvers such as CVXOPT, GLPK, CyLP (a Python interface to CBC), and Gurobi Optimizer. These solvers generally use the simplex method or interior point method to solve the continuous linear programmings and branch and bound method to solve MILP formulation. In general, the continuous linear programming is solved much faster due to the fact that optimal solution can always occur at corner points in the feasible region.

I found the Gurobi Optimizer the fastest one to work with, also, it has many tuning parameters which may help in special cases. All these solvers are implemented in C and are fairly fast in terms of the programming. The main difference is due to the algorithms they use, and the way they manage the multicores and parallel calculation (using Gurobi, we can easily manage the number of cores). Finally, we use their Python interface which makes the formulation much easier, as most of them provide model objects to work with. 


\section{Chapter 4}

\section{Millimeter Wave Access Network \\ Planning}

\subsection{Introduction}

Higher required data rates and bandwidth shortage in the currently utilized spectrum drives the next generation of wireless networks to move forward to higher carrier frequencies, especially, mmWaves, which have attracted much attention during the past few years $[1,2,59]$. mmWave band, which is nominally considered between $30 \mathrm{GHz}$ to $300 \mathrm{GHz}$, can increase wireless networks bandwidth by an order of magnitude.

However, mmWave propagation characteristics are qualitatively different than those of sub-6 GHz waves. The major differences are as follows: first, they are more sensitive to blockage [22]; as a result of mmWave smaller wavelength, there is a severe penetration loss when passing through many common materials such as concrete and tinted glasses. The loss from a $3.8 \mathrm{~cm}$ tinted glass (typical exterior surface of urban buildings) might be as high as $40 \mathrm{~dB}$ [2]. Also, as the wavelength is much smaller than city dimensions, diffraction is low, and the waves usually can not diffract over the corners and propagate from one street to another. The mentioned limitations 
magnify the LOS need for communications.

Second, the antenna effective area is proportional to wavelength square, resulting in a higher path loss. According to the Friss equation, increasing frequency results in $20 \mathrm{~dB}$ per decade more path loss [59]. Two approaches are usually considered dealing with the mmWave high path loss. First, cell radiuses should be smaller, and second, there should be some degree of beamforming to concentrate the power.

The above discussion brings the idea that short-distance LOS communication is the preferable theme in mmWave wireless networks. Also, owing to smaller wavelengths, BS dimensions are much smaller than traditional tower BSs. These two properties dramatically change the way a network should be planned, because the LOS small cells are highly affected by the city layout. There are many opportunities in an urban area to place the BSs, such as lamp posts, traffic lights, above-rooftop small BSs, and wall-attached BSs. We use the wall-mounted BS option, because the walls data can be readily found from open source maps. The network planning aims to decrease the cost by reducing the number of BSs deployed in a particular area.

In this chapter, we plan the outdoor wireless access networks using wall-mounted BSs, with the goal of minimizing the number of BSs to provide the LOS coverage. We use computational geometry to model the problem and we use optimization tools to find the BS places. At the end of this chapter, we give some useful simulation results regarding the BS densities (number of BSs per $\mathrm{km}^{2}$ ) required for the outdoor LOS coverage networks. Also, we analyze some network properties such as noise or interference limitations using the planned network. 


\subsection{Problem Statement}

In this thesis, we only consider two-dimensional network planning and we add no constraints on the height of BSs. However, to avoid obstacle blockage in the street, higher BSs are preferred [40]. As long distance communication is more difficult we force the maximum cell radius to be $200 \mathrm{~m}$, where the LOS communication is usually considered to be feasible in this range in dense urban areas [2,22]. We only consider the LOS coverage, and if an area does not have the LOS access it is considered as an outage. Finally, we use real maps to find some understanding about the possible BS densities required in urban areas, we use OpenStreetMap project as our real maps database. Also, to consider different city layouts effect in a mmWave network, we run the simulations over two metropolitan area, one in Manhattan Island, and one in central Paris.

Our proposed network planning method is divided into two steps, first, we place many candidate locations on the map, and then we select a small subset of candidate locations to maximize the LOS coverage. The candidate placement algorithm is based on the [13] and is described in more detail in Section 4.3.

After placing the candidate locations, we select a small subset of them to maximize the coverage. First, we develop a greedy approach to approximate the optimal solution. The greedy approach needs less computational cost and scales easier for larger maps. Second, in Section 4.4.2 we propose a binary linear optimization programming (BILP) formulation to maximize the coverage. Finally, in Section 4.4.3 we relax the integer and binary constraints in optimization formulation to find an upper bound on the coverage. This upper bound is useful to analyze the greedy performance in larger maps, since it is a simple linear programming (LP) and scales easier.

In this thesis, we use real map building footprints data instead of probabilistic 


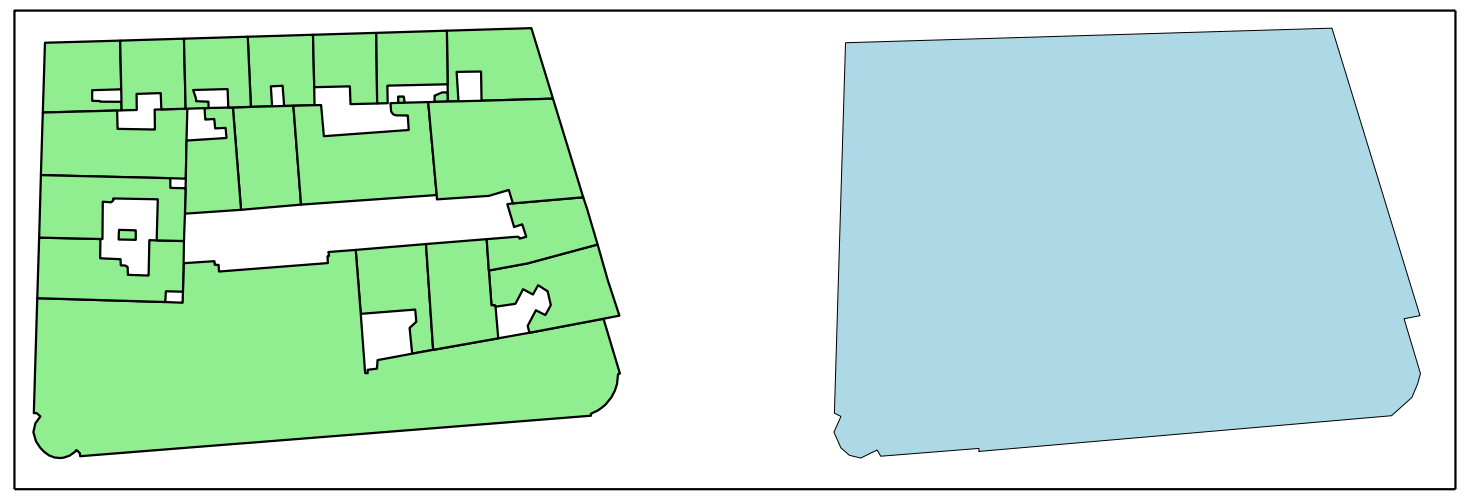

Figure 4.1: An example of how adjacent buildings are merged together and holes inside them are removed to create a simple polygon.

blockage modelling. The reason is that one of the contributions of this thesis is to find representative simulation results regarding the BSs densities, which requires detailed enough models. The major contribution is to estimate the number of BS required for mmWave networks. BSs density is an important network parameter as it affects the properties of network such as interference and noise limitations as well as the deployment cost. We gathered the buildings' footprints from OpenStreetMap project as the maps are free to access, accurate, and frequently updated [18].

\subsection{Candidate Location Placement}

The candidate placement is based on the algorithm in [13]. In this section, we give a description of the way it works. The main idea is to find the best place on each side of a building. The algorithm consists of three major steps within itself which we describe in following paragraphs.

It first extracts the buildings from the database, then it merges the adjacent buildings and removes the holes inside them to create simple polygons (SPs). Fig. 4.1 shows the result of the first step. 
Then it finds the natural neighbors of each SP. If the buildings are placed in a regular grid manner, finding their neighbors is obvious, but in a real city, buildings can be anywhere, so a more general method is needed. The algorithm uses the generalized Voronoi diagram, which is as follows: for each SP, it assigns a cell, where the cell contains the SP and all the points on the map which are closer to that SP than any other SP. The resulting cells create the generalized Voronoi diagram. Then, any two cells which touch each other are referred as natural neighbours.

After finding the natural neighbours of each SP, it partitions each SP's contour as follows: for any pair of natural neighbours it finds the closest two points, so as to create the shortest link between these two SPs. Then, these points on each SP

partition its contour. After partitioning the contour of each SP, each segment is searched to find the point with the maximum LOS area, and that point is chosen as a candidate location. The result is shown in Fig. 4.2.

\subsection{Maximizing the Coverage}

After placing the candidate locations, our goal is to select a small subset of them and maximize the LOS coverage. The problem is to choose a $K$-subset of candidate locations to maximize their total coverage. First, to model the coverage, we distribute points on uniform dense grid in the outdoor area. Fig. 4.3 shows these grid points. Then, we create the binary matrix $C=\left\{c_{i j}\right\}$ to contain the joint coverage information of candidate locations, that is $c_{i j}$ is 1 if and only if candidate location $i$ covers point j. We use matrix $C$ in our further calculations.

We have two different approaches, which we outline in following two subsections. 


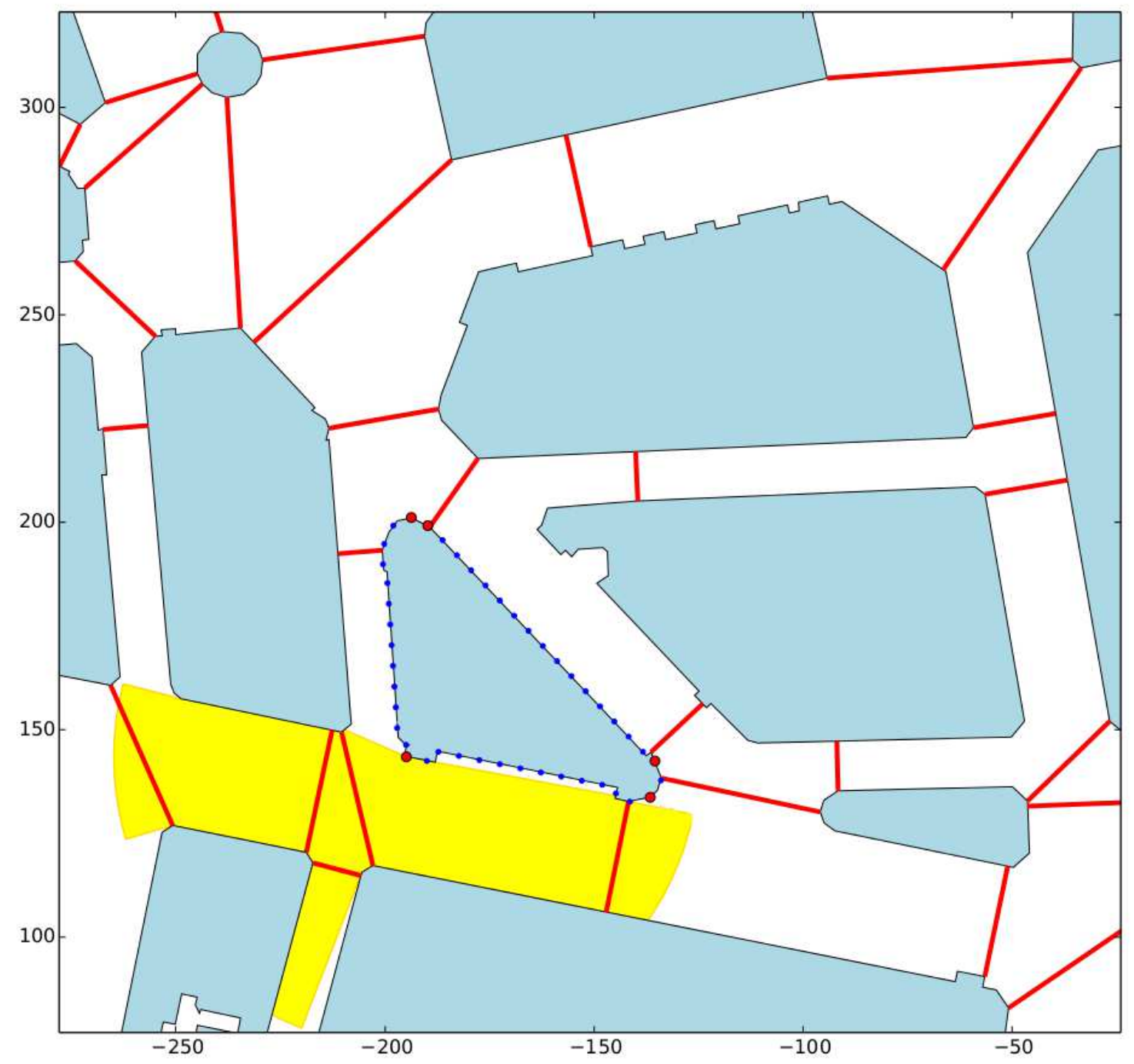

Figure 4.2: Candidate location placement. The red lines are the shortest links between natural neighbour simple polygons. Candidate locations are found by searching each segment in the simple polygon for the point with largest line-ofsight coverage area. That is to say, each candidate location has a local maximum line-of-sight coverage. 




Figure 4.3: Result of candidate location placement algorithm with distributed points. To model the coverage, we distribute grid points on the map and create binary matrix $C$, where each candidate location takes one row in the matrix and each point one column. Coverage area of one example candidate location is indicated by yellow area. 
Section 4.4.1 gives a heuristic method using greedy addition and Section 4.4.2 introduces a BILP formulation to find the optimal solution. Finally, in Section 4.4.3 we relax the binary integer constraints to provide an upper bound on the LOS coverage area.

\subsubsection{Greedy Addition}

We use real maps in our simulations, therefore, the size and the shapes of these maps are formed based on real situations and they can be widely different. As a result finding the optimal solution might be extremely time-consuming in some situations. Therefore, we approximate the optimal solution first. Although greedy addition is not going to give us the optimal, it is highly useful when we are simulating larger maps.

The main idea is to divide the problem into steps, and in each step choose the best candidate location, then update the points to be covered and repeat all these $K$ times, where $K$ is the desired number of ABSs to select which we sweep it to find the required ABS density for practical coverage percentages. The greedy addition can be formulated as follows:

- If $K$ is zero, end.

- Choose the best candidate location (one that covers the largest number of points).

- Remove all of the points covered by selected candidate locations.

- Assign $K \leftarrow K-1$ and go to the beginning.

The greedy addition needs more candidate locations to cover the same amount of area than the optimal algorithm, but its coverage area is guaranteed to be at least 
$(1-1 / e) \approx 0.63$ of the optimal solution's area with the same number of candidate locations [60]. The greedy addition is a polynomial-time problem and it is shown in [61] to be the best polynomial-time approximation for finding the solution of the maximum coverage problem. Moreover, the greedy addition is simple to implement and scales easily for larger maps.

\subsubsection{Binary Integer Linear Programming}

In this section, we introduce a BILP formulation to find the optimal solution. We have already discretely modelled the problem by distributing grid points in the map, therefore the coverage problem transforms to maximizing the number of covered points. Assuming that there are $m$ points in total in the outdoor area, we create the binary variable $P=\left\{p_{i}\right\}_{1 \times m}$ to decide whether a point is covered or not. That is to say, $p_{i}$ is 1 if point $i$ is covered. Having $n$ candidate locations, to decide which candidate location to select we create the binary variable $B=\left\{b_{i}\right\}_{1 \times n}$, where $b_{i}$ is 1 if and only if candidate location $i$ is selected. By having the binary variables $B$ and $P$ and the binary matrix $C$ defined at the beginning of this section, the LP formulation is stated as follows:

$$
\begin{array}{rlr}
\text { maximize } & \sum_{j=1}^{m} p_{j}, & \\
\text { subject to } & \sum_{i=1}^{n} c_{i j} b_{i} \geq p_{j}, & \\
& \sum_{i=1}^{n} b_{i}=K, & \\
& p_{j} \in\{0,1\}, & \\
& b_{i} \in\{0,1\}, & j \in\{1, \ldots, m\}, \\
& &
\end{array}
$$


where the objective function 4.1 comes from the fact that we want to maximize the coverage. The set of $m$ constraints in 4.2 ensures that if a point is covered $\left(p_{j}=1\right)$, at least one candidate location covering that point should be selected. And the constraint 4.3 states the fact that we want to select $K$ candidate locations in total.

This formulation results in the optimal solution, which means there is no better $K$-subset of candidate locations that covers a larger number of points. However, this formulation is NP-hard [61], and solving it is computationally costly compared to the greedy addition method. In particular, it takes more time for larger maps, and does not scale easily.

\subsubsection{Upper Bound on Coverage}

Since the maximum coverage problem is modelled as BILP, it may be hard and timeconsuming to find the optimal solution. In this section, we maximize the objective function while we relax the binary integer constraints to continuous constraints. This results in the following formulation:

$$
\begin{aligned}
& \operatorname{maximize} \sum_{j=1}^{m} p_{j} \\
& \text { subject to } \sum_{i=1}^{n} c_{i j} b_{i} \geq p_{j}, \quad j \in\{1, . ., m\} \text {, } \\
& \sum_{i=1}^{n} b_{i}=K \\
& 0 \leq p_{j} \leq 1, \quad j \in\{1, \ldots, m\}, \\
& 0 \leq b_{i} \leq 1, \quad i \in\{1, \ldots, n\},
\end{aligned}
$$

where all the parameters are the same as the formulation in Section 4.4.2.

In most cases, the values of $P$ and $B$ are fractional and the result is not feasible 
to the original solution [62]. However, as the new region includes the feasible set, the founded objective function can be considered as an upper bound for the problem. The bound is useful to analyze the greedy addition performance when BILP is too costly. In contrast to the BILP, which is an NP-hard problem in general, this formulation can be solved in polynomial time.

\subsection{Simulation and Results}

We use the candidate placement algorithm as described in Section 4.3 as the first step. Then, we plan the network using the formulations introduced in Section 4.4 to provide the LOS coverage. Mainly, we run our simulations over real maps, where the map information is summarized in Table 4.1. But to make the simulations more easily reproducible, we run the simulations on the Manhattan grid map, shown in Fig. 4.4. In Manhattan grid map the street width is set to $20 \mathrm{~m}$ and buildings are rectangles with $50 \mathrm{~m} \times 150 \mathrm{~m}$ dimensions, placed parallel to each other. We place candidates on each corner and on each rectangle side center to include the effect.

Then we provide simulation results about some important properties of the wireless networks, such as density of BSs, statistics of the line of sight portion of a cell, and the number of possible strong interferences. Also, more importantly, we compare two different dense urban areas according to the shape of the streets.

\subsubsection{Density of BSs for Different LOS Coverage Percentages}

Dense BS deployment is a possible theme in next-generation cellular networks. The BSs density affects many properties of the network, and directly affects the deployment, maintenance, and hardware setup costs. Having a general idea about the 


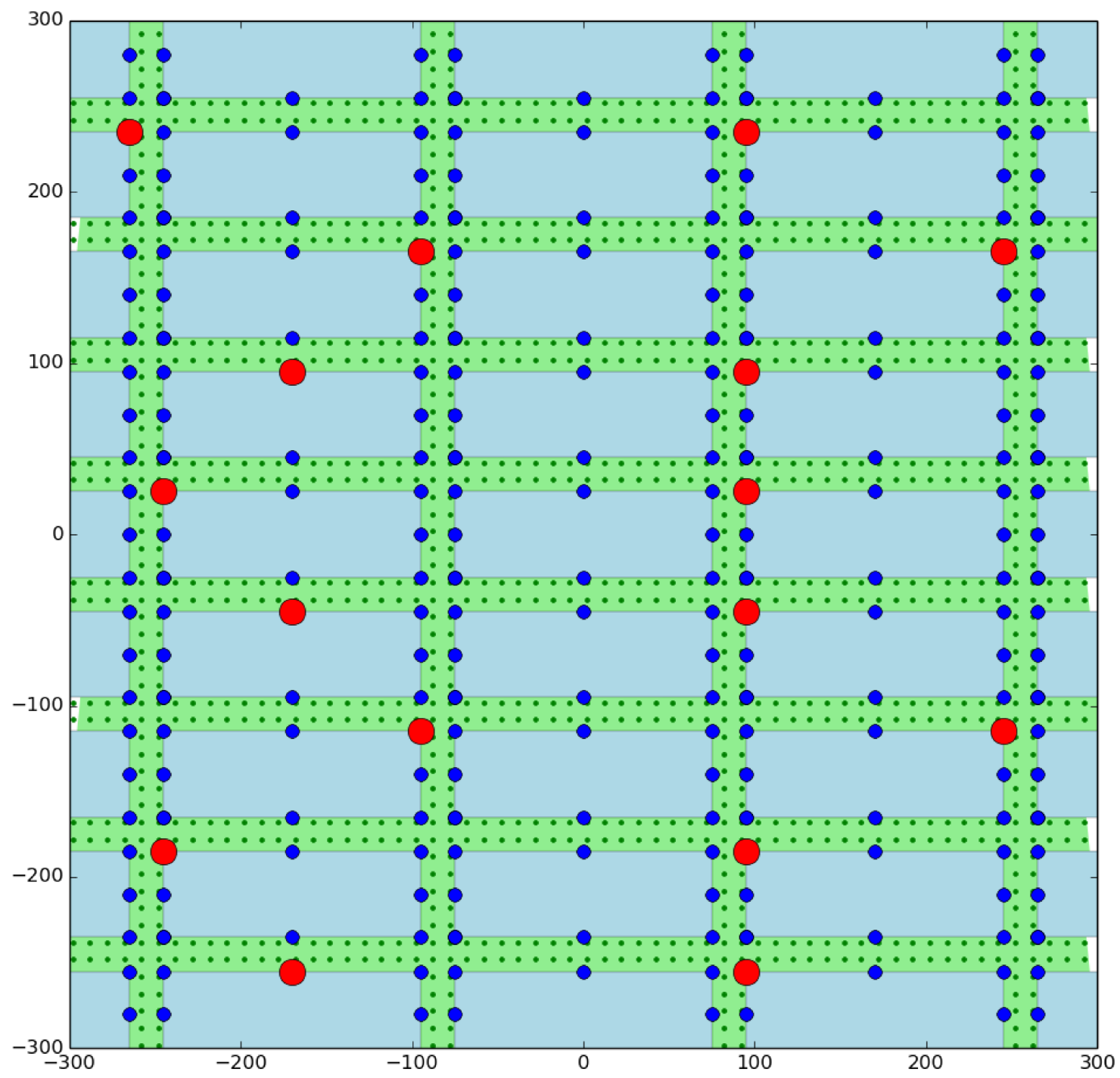

Figure 4.4: Manhattan grid map. It is designed to be similar to highly dense urban areas where we used $20 \mathrm{~m}, 150 \mathrm{~m}$, and $50 \mathrm{~m}$ for street width, building footprints' width and height, respectively. Candidate locations are indicated with blue circles, where we placed them on building corners as well as the middle of each side. Selected candidates are shown in red, where the result is find by running the binary integer linear programming with $K$ set to 16 . 


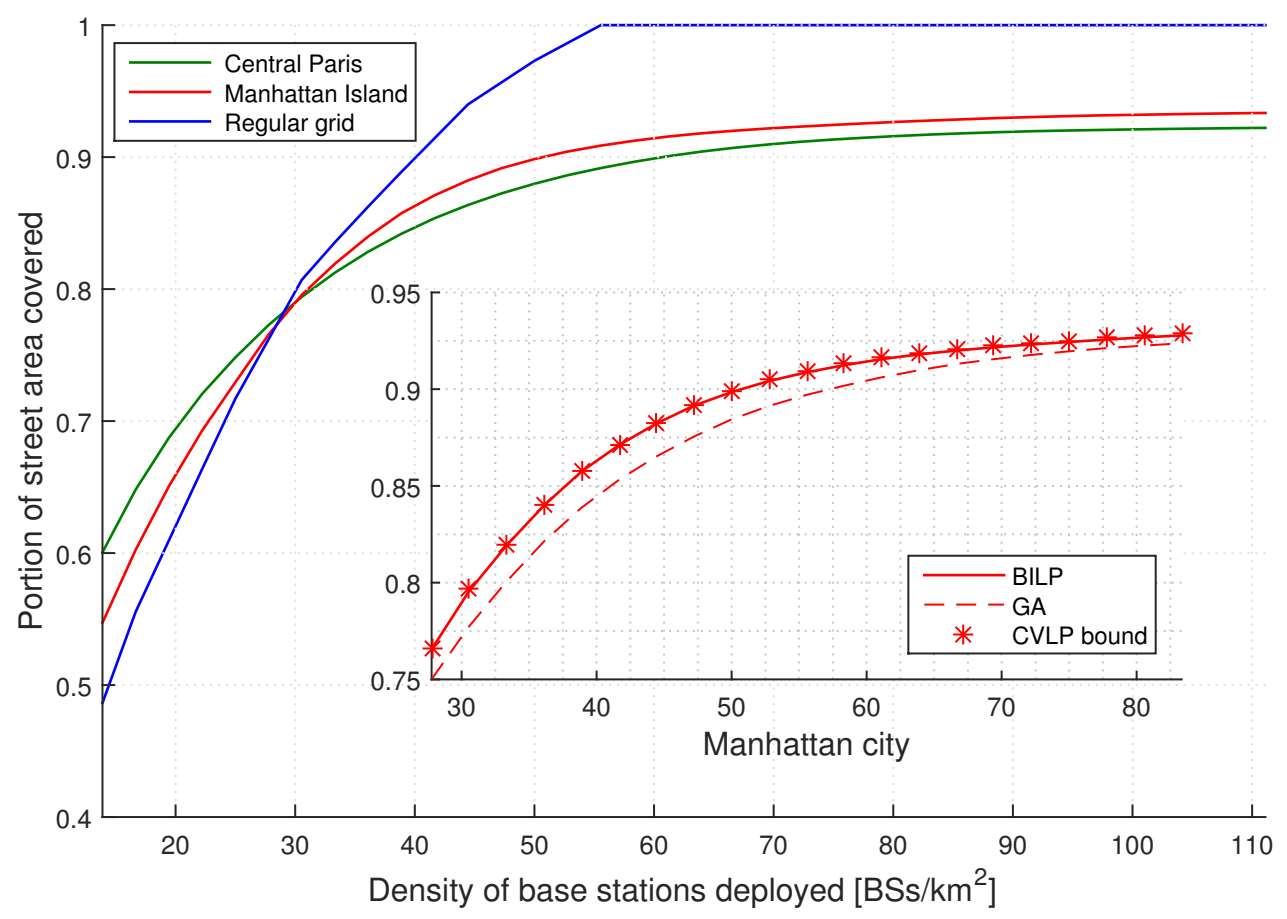

Figure 4.5: Base station densities required for different outdoor line-of-sight coverge fraction. The figure shows the simulation results for different urban areas, as well as the Manhattan grid map. we found 40, 50 and 60 base station per $\mathrm{km}^{2}$ densities to cover 90 percent of outdoor area. Also, the inner figure compares the differences between the greedy addition, binary integer linear programming and the bound.

possible BS densities is essential for a network designer. In this section, we find the densities required for the different LOS coverages, based on our planning method. The results are found by running the planner over 12 and 17 maps from Manhattan Island and central Paris and taking the average over all of them. That is, to sweep the values of $K$ from 0 to 100 BSs per $\mathrm{km}^{2}$ and record the ratio of the number of points covered and the total number of points distributed in the outdoor area. Fig. 4.5 shows the results.

We found that around 40, 50 and $60 \mathrm{BSs}$ per $\mathrm{km}^{2}$ are needed to cover 90 percent 
of the outdoor area in the Manhattan grid, Manhattan Island, and central Paris, respectively. The different densities are the result of differences in city layouts, whereby Manhattan streets are straight and long, while the bending streets reduce the chance to have long LOS links. Also, on average, the greedy addition places 16 percent more BSs than optimal. We can see that the bound is tight, as its maximum difference with the optimal solution shows less than 4 percent more density.

\subsubsection{Probability Distribution of Cell's LOS Fraction}

One other useful piece of information lies in the portion of the LOS coverage in the cell. That is to say, if a cell is placed properly, there is an increased probability of greater line of sight area. After we plan the network, we calculate the cumulative density function of the fraction of LOS and give its mean and variance in Table 4.1.

The LOS coverage results can be used together with path loss models to calculate link budgets and rate coverage for outdoor urban areas.

\subsubsection{Noise and Interference Analysis in Millimeter Wave Networks}

Finally, one important feature of any network is its power analysis. In this section, we calculate the signal-to-interference-plus-noise ratio (SINR) in our planned map. As we plan the network to establish LOS coverage, there are some parts in the map where different cells overlap. Fig. 4.7 shows the number of times an area is covered in the map. The results indicate that each area is covered an average of 1.4 times, which means that in dense urban BS deployment, there is one strong interference link almost half of the times. Which as we have wide spectrum space in mmWave one can use some frequency reuse pattern or wider bandwidth that allows for automatic 




Figure 4.6: Cumulative density function of the line-of-sight fraction of a cell for different cell radiuses. When we consider $200 \mathrm{~m}$ cell radiuses, the probability of having more than 20 percent line of sight area in a cell drops to less than 10 percent. Also, the curves saturate at around 75 percent due to the fact that we are placing the base stations on wall corners, meaning that there is always 25 percent blocked by the mounted building. 


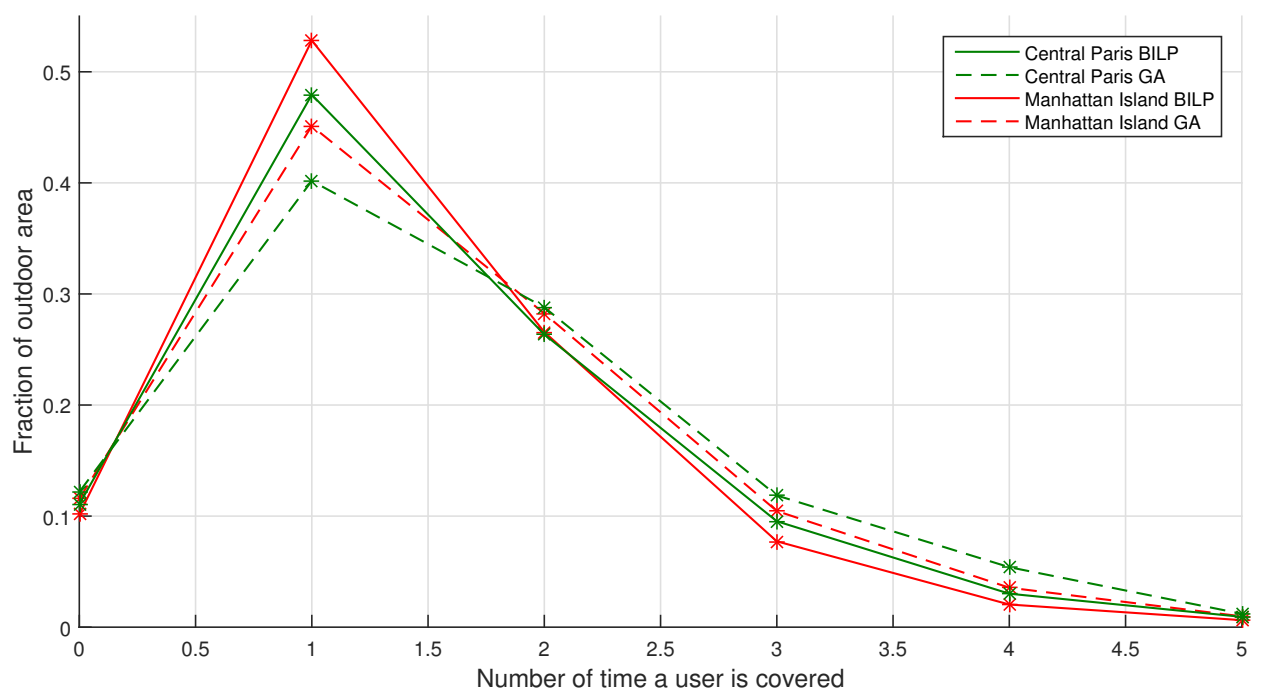

Figure 4.7: Number of times a certain area is covered, for 90 percent outdoor area coverage. A typical outdoor user is covered by about 1.4 base stations on average, meaning that the number of strong interferers (both line-of-sight and within $200 \mathrm{~m}$ ) is about 0.5 on average. There are almost never more than 3 strong interferers

coordination.

The mmWave channel modelling is an ongoing research area and the NLOS propagation is not completely known. However, several measurement campaigns done in $[63,64]$, have gained attention in the past few years. As LOS and NLOS propagation may have different path-loss characteristics, to find received power at a particular point, we first find out whether or not it is in the LOS area of a BS. Following that, we pick the appropriate channel model based on [26]. Finally, we calculate SINR, SNR, and SIR distributions in our planned maps. We consider the areas which do not have LOS connection with any BS in outage.

We use the same channel model as in [26], where the authors use measurement data values of $28 \mathrm{GHz}$ and set the parameters of the standard linear model, 


$$
P_{L}(d)[d B]=\alpha+10 \beta \log _{10}(d)+X, \quad X \sim N\left(0, \sigma^{2}\right),
$$

where $P_{L}$ is the path loss in $\mathrm{dB}, d$ is the distance from the transmitter, $\alpha$ and $\beta$ are the linear tuning parameter sets, and $X$ is a zero mean Gaussian random variable with variance $\sigma^{2}$. We use different parameter values for line of sight and NLOS scenarios. The values are given in Table 4.1

We further assume the transmit power is $30 \mathrm{dBm}$ and the serving link antenna gain is $16 \mathrm{dBi}$, which are more conservative values compared to the ones assumed in [12]. Furthermore, we set all interfering antenna gains (all BSs except the serving one) to be $5 \mathrm{dBi}$. Now we can calculate SINR as follows:

$$
S I N R=\frac{P_{t x} G_{s} / P_{L}\left(d_{0}\right)}{\sum_{k} P_{t x} G_{i} / P_{L}\left(d_{k}\right)+W N F},
$$

where $G_{s}$ and $G_{i}$ are the serving and interfering antenna gains, respectively; $W$ is downlink bandwidth; $N$ is noise power density; and $F$ is the noise figure. Also, to find the distance $(d)$, we calculate the 3D distance with the transmitter and receiver heights set to $15 \mathrm{~m}$ and $1.5 \mathrm{~m}$, respectively.

A fundamental question for the design of mmWave networks is to understand the effects of interference and, more specifically, the likelihood that mmWave wireless networks will be limited by interference or by thermal noise. For example, while interference-limited networks can benefit from advanced techniques such as intercellular interference coordination, coordinated beamforming and dynamic orthogonalization, these techniques have little value in networks where thermal noise, rather 
Table 4.1: Simulation Setup and Results Summary

\begin{tabular}{|l|l|l|}
\hline Urban area & South Manhattan & Central Paris \\
\hline Centre & $73.9865^{\circ} W, 40.7468^{\circ} N$ & $2.334^{\circ} E, 48.8600^{\circ} N$ \\
Number of maps & $2 \times 5$ & $7 \times 4$ \\
Map size & $1 \mathrm{~km} \times 1 \mathrm{~km}$ & $1 \mathrm{~km} \times 1 \mathrm{~km}$ \\
Area studied & $600 \mathrm{~m} \times 600 \mathrm{~m}$ & $600 \mathrm{~m} \times 600 \mathrm{~m}$ \\
User grid spacing & $10 \mathrm{~m}$ & $10 \mathrm{~m}$ \\
\hline LOS area within & & \\
$50 \mathrm{~m}$ & $\hat{\mu}=48.3 \%, \hat{\sigma}=11.7 \%$ & $\hat{\mu}=33.6 \%, \hat{\sigma}=14.7 \%$ \\
$100 \mathrm{~m}$ & $\hat{\mu}=28.1 \%, \hat{\sigma}=9.9 \%$ & $\hat{\mu}=19.1 \%, \hat{\sigma}=12.4 \%$ \\
$150 \mathrm{~m}$ & $\hat{\mu}=19.0 \%, \hat{\sigma}=8.3 \%$ & $\hat{\mu}=12.8 \%, \hat{\sigma}=10.3 \%$ \\
$200 \mathrm{~m}$ & $\hat{\mu}=14.1 \%, \hat{\sigma}=6.8 \%$ & $\hat{\mu}=9.37 \%, \hat{\sigma}=8.63 \%$ \\
\hline Parameters & Meaning & Value \\
\hline$P_{t x}$ & transmit power & $30 \mathrm{dBm}$ \\
$G_{S}$ & serving link antenna gain & $16 \mathrm{dBi}$ \\
$G_{i}$ & interfering link antenna gain & $5 \mathrm{dBi}$ \\
$W$ & downlink bandwidth & $500 \mathrm{MHz}$ \\
$N_{0}$ & thermal noise & $-174 \mathrm{dBm} / \mathrm{Hz}$ \\
$F$ & noise figure & $7 \mathrm{~dB}$ \\
$L_{L}(d)$ & LOS path loss & $61.4+20.0 \log _{10}(d) \mathrm{dB}$ \\
$L_{N}(d)$ & non-LOS path loss & $72.2+29.2 \log _{10}(d) \mathrm{dB}$ \\
$h_{\mathrm{BS}}-h_{\mathrm{M}}$ & propagation height difference & $15 \mathrm{~m}-1.5 \mathrm{~m}$ \\
\hline
\end{tabular}


than interference, is dominant [65].

One important factor which affects the network's interference limitations considerably is the density of BSs. To evaluate the proposed network planning method, we find the SINR, SNR, and SIR distributions, shown in Fig. 4.8.

Finally, Fig. 4.9 summarizes the SINR values in the map. As can be seen, the values are shaped by the city geometry, which indicates again the gain one can get by planning the network, and BS locations. 

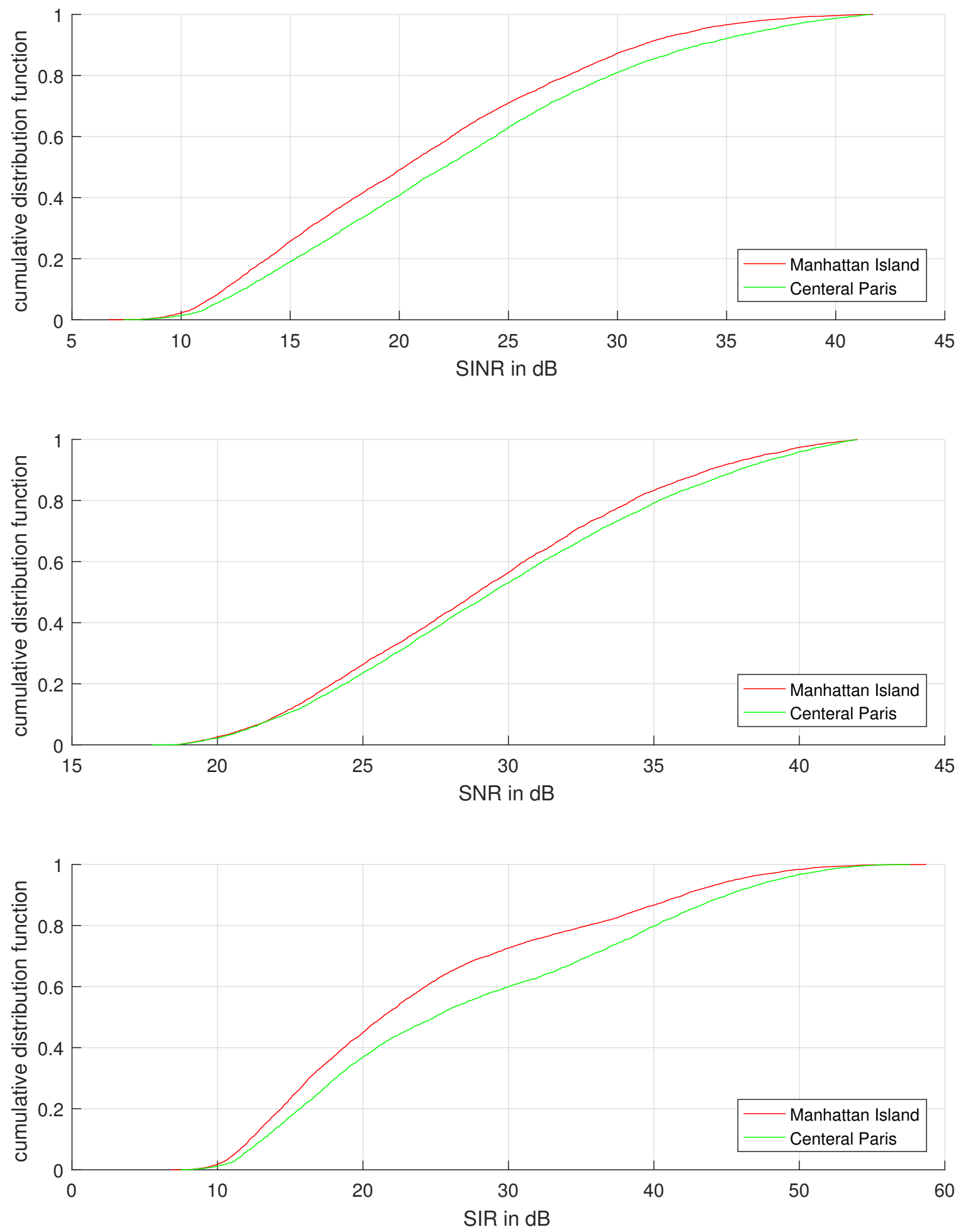

Figure 4.8: Power distribution in planned maps. A large fraction of the outdoor area is covered with SINR of more than $20 \mathrm{~dB}$. The figure suggests that low SINR is mainly caused by interference, and the SINR curve is more similar to the SIR curve for small SINR values (less than $20 \mathrm{~dB}$ ), and it is more similar to the SNR curve for larger SINR values (more than $35 \mathrm{~dB}$ ). 


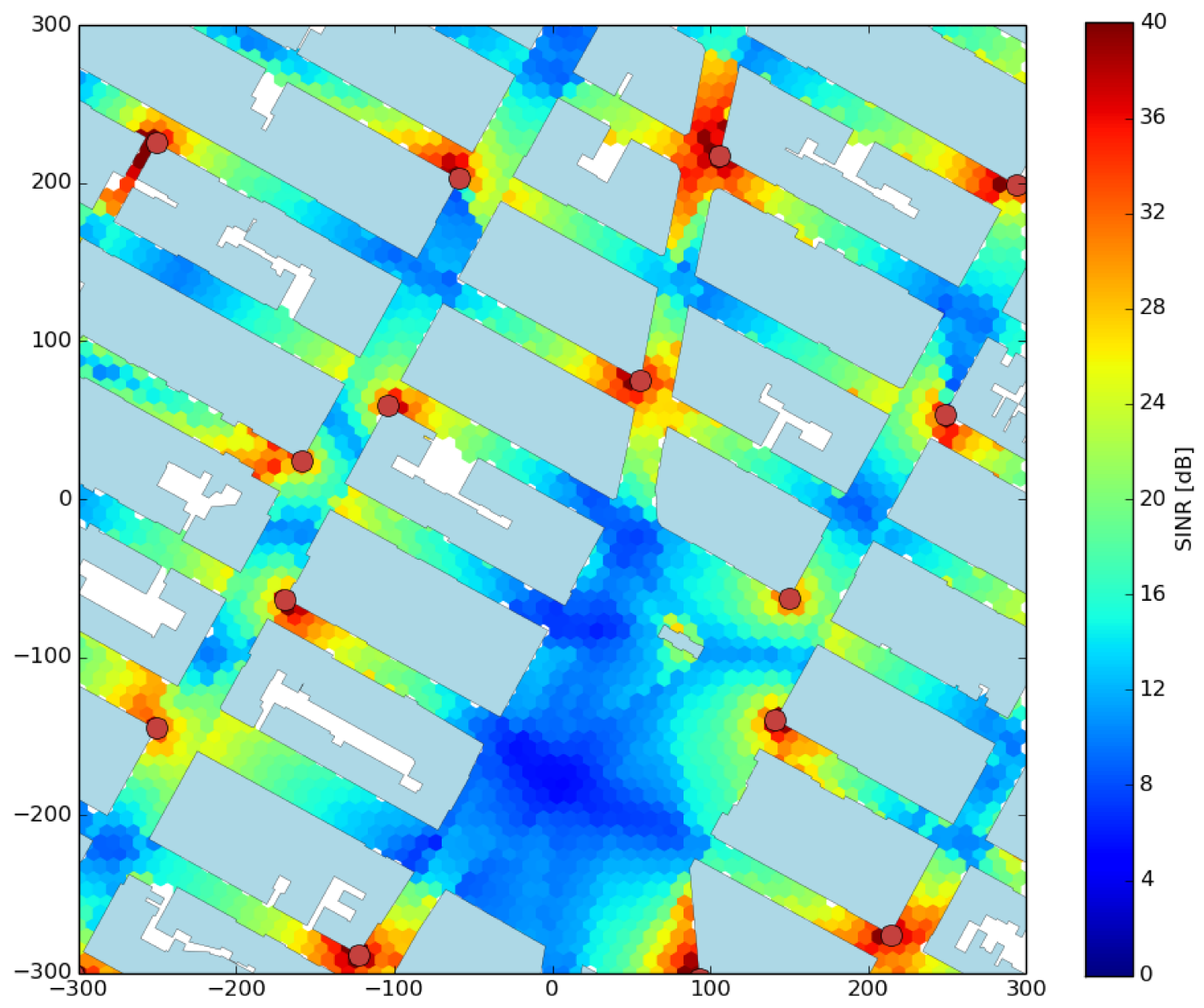

Figure 4.9: Result of the binary integer linear programming with 50 base stations per $\mathrm{km}^{2}$ in Manhattan Island area, with its corresponding spatial SINR distribution. White regions do not have a serving LOS link and are considered in outage. 


\section{Chapter 5}

\section{Millimeter Wave Backhaul Network Planning}

\subsection{Introduction}

Wireless data streaming expansion, critical applications and increased number of connected devices are pushing the telecommunications community to move forward to the next-generation wireless networks. Future networks are predicted to require much higher data rates, lower latencies, and more energy and cost efficiency [1]. An important solution to increasing the data rate is to increase the bandwidth. As the currently utilized ultra high frequency (UHF) bands are congested, a shift to higher carrier frequencies is predicted. The mmWave bands can provide much larger bandwidth and are considered as the main candidate band for 5G networks [2]. Using mmWaves brings several challenges, as they are more sensitive to blockage, they are

not diffracted easily and they have higher path loss. To compensate for blockage sensitivity, LOS communication is preferred. Beamforming is also a potential solution to counter high path loss.

In current sub-6 GHz networks as well as future networks, one way to approach 
the high demand for capacity and high end-user data rate requirements could be to increase the number of cells. That is, to deploy cells with shorter inter-site distances [29]. Dense BS deployment is one way to decrease the number of users per cell and, consequently to assign larger band width resources to each user. Another useful way to decrease the number of users per cell is to have cells with smaller radiuses and to communicate over shorter distances, which increases the recieved signal power and spectral efficiency [30]. The main disadvantage simply arives from the fact that deployment of large numbers of BSs is too costly, and bringing fiber connections to very each of these cells might be infeasible.

The major solution to overcomming high fiber connection costs in dense networks is to connect only a few of the BSs to the core network via fiber and connect the rest of the BSs via wireless backhaul links. Due to new advancements in physical layer technologies such as hybrid beamforming and full duplexing [34], mmWaves can provide high capacity links between access BSs (ABS), and can be considered as a potential enabler technology for small cell deployment [3]. Backhaul links should aggregate all users' data to the wired connected BSs (gateways), meaning that, backhaul links should be high capacity links to ensure reliable communication. The mmWaves are going to be used in access links and each user should be able to consume a considerable amount of bandwidth. As such, larger bandwidths should be used in backhaul to include all users' collected data. As a result, the main candidate backhaul communication scenario would be the LOS mmWave communication [3].

In this paper, we focus on wireless backhaul opportunities, particularly, mmWave LOS links, and we plan the wireless access and backhaul networks using wall-mounted BSs. We introduce a mixed integer linear programming (MILP) formulation to find the ABS locations. Our objective is to maximize the coverage and, importantly, we force the ABSs to see each other. Then we place the wired-connected ABSs, 
referred to as gateways, to minimize their total number. We run the simulations over two real dense urban areas, which are the probable areas to implement nextgeneration wireless networks, and found simulation results regarding the ABS and gateway densities required for the LOS coverage. Also, we calculate the empirical distribution of backhaul-links distances.

\subsection{System Model and Problem Statement}

\subsubsection{Fresnel Zones}

The quality of backhaul links is crucially important in wireless networks, as they directly affect the reliability, latency, and efficiency of the network. If there are two base stations placed on the same wall, there will be a line-segment (optical) connection between them. However, we can intuitively state that their optical link is a poor link, especially when this will be used for backhaul.

The line of sight communication is usually considered feasible if approximately 60 percent of the first Fresnel zone is not disturbed. In this case, free-space path loss is a good channel model to use [32]. The Fresnel zone radius at point P between the end points of a link is given as follows:

$$
F_{n}=\sqrt{\frac{n c d_{1} d_{2}}{f\left(d_{1}+d_{2}\right)}}, \quad \max \left(F_{1}\right)=\frac{1}{2} \sqrt{\frac{c D}{f}},
$$

where $f$ is the wave frequency, $c$ is the speed of light, $d_{1}$ and $d_{2}$ are the distances between $\mathrm{P}$ and link end points, and $D$ is the total link distance [59].

We assert two points see each other if their first Fresnel zone is not disturbed. The reason why we do not consider line-segment (optical) line of sight is that, although 




Figure 5.1: Fresnel zones approximation. We approximate the Fresnel zones with a diagonal shap. The figure shows the first and 10th Fresnel zones in dark and light green, respectively. As can be seen, in millimeter wave frequencies, the maximum first Fresnel zone radius is much smaller than building dimensions.

access base stations placed on adjacent walls can connect to each other with a linesegment, about 50 percent of their first Fresnel zone is blocked. We use a $28 \mathrm{GHz}$ frequency to find whether two points see each other or not. Furthermore, To make the calculation easier, we approximate the ellipse with a diamond, with $D$ and $\max \left(F_{1}\right)$ as its larger and smaller diameters. For $f \geq 28 \mathrm{GHz}$ and $D \leq 400 \mathrm{~m}$ we have $\max \left(F_{1}\right) \leq 1.07 \mathrm{~m}$, which indicates the narrowness of millimeter waves' first Fresnel zone compared to building dimensions, as can be seen in Fig. 5.1

\subsubsection{Engineering Setup}

The goal of this chapter is to plan the wireless backhaul and access network for the outdoor area. We only consider the outdoor area, as the outdoor and indoor networks are isolated from each other due to high penetration loss [2]. Also, we consider only 2D network planning, but higher BS locations provide better coverages due to low-altitude obstacle blockages in the streets. Because of high path loss in high frequencies, we limit the maximum access link distance to $200 \mathrm{~m}$, as in [2]. Also, we set the maximum backhaul link distance to be $400 \mathrm{~m}$, as there are more gains on the 




Figure 5.2: Network planner steps. Candidate location placement is briefly described in Section 4.3. Access base station and selection and gateway placement algorithms are described in Section 5.3 and Section 5.4, respectively.

receiver side, and BSs are possibly fixed. As the authors state in [3], there is possible backhaul communication for distances up to $1 \mathrm{~km}$. At the end of this chapter, we also give the empirical distribution of backhaul link distances from the planned networks. Finally, we use OpenStreetMap data base for real maps.

The entire algorithm is divided into three main steps: first, we place many candidate locations on the map, where each one is found by locally searching the contour of the buildings. The candidate placement algorithm is described in more detail in Section 4.3. After placing the candidates, we select a small subset to maximize the coverage, while the selected candidates create a connected graph themselves. Then, among the selected candidate locations, we choose ones to install fiber such that their total number is minimized. The selected candidate locations are our planned ABSs, and the fiber-connected BSs are referred to as gateways in this thesis.

To summarize the candidate location information, we create the graph $G_{0}$ such that each candidate location is one $G_{0}$ node and there is an edge between two $G_{0}$ nodes if and only if the corresponding candidate locations see each other. The problem is therefore to select $K$ nodes from the $G_{0}$ (a $K$ subgraph) such that the resulting graph is connected. The resulting subgraph includes the ABS places and is referred to as $G_{1}$ in this chapter. Having $G_{1}$, we then place gateways on the nodes to minimize the total number of gateways. The steps are shown in Fig. 5.2. 


\subsection{Maximizing the Coverage with Connectivity}

An efficient mmWave network provides coverage with a small number of ABSs. One can only maximize the coverage using the maximum coverage problem as done in [7]; however, as we aim to plan a wireless backhaul network, we need to consider further requirements in ABS planning. To make wireless LOS backhaul possible we plan the network in a way that ABSs see each other. That is to select candidates such that $G_{1}$ be a connected graph. Planning the network in this way ensures ABSs do not become isolated. Moreover, if the allowable number of hops (the number of ABSs between the user and the gateway) increases, the introduced planning method can be used without any changes. In fact, if there is no limitation on the number of hops, creating a connected graph of ABSs will need only one gateway. In that case, this approach is optimal and maximizes the coverage with $K$ ABSs and 1 gateway.

As stated in 4.3, we first place many candidates on the map and create the graph $G_{0}$, which has all the information we need regarding the candidate locations. Each $G_{0}$ node is a candidate location and there is an edge between two nodes if they see each other. Furthermore, to model the joint coverage area of the candidate locations, we distribute points on a small square grid area in the map. Having $n$ candidate locations and $m$ points on the map, we create the joint coverage binary matrix of candidate locations called $C=\left\{c_{i j}\right\}_{n \times m}$, where each row stands for one candidate location, each column stands for one point, and $c_{i j}$ is 1 if and only if point $j$ is in the LOS coverage area of candidate location $i$. We use matrix $C$ in the following formulations.

In this section, we plan the ABSs such that they create a connected graph. Actually, this problem is a variation of tha art gallery problem, called the art gallery problem with cooperative guards [66]. The art gallery is described in more detail in 
Section 3.2. Here we introduce a MILP formulation based one the connected subgraph problem [67]. Also, we develop a greedy approach to approximate the optimal solution and at the end of this section, we provide an upper bound on the optimal solution by relaxing some of the constraints in the optimization formulation. In this context, we call this formulation the maximum coverage problem with connectivity (MCPC).

\subsubsection{Mixed Integer Linear Programming}

To find the optimal solution we introduce a network optimization formulation similar to the approach taken by the authors in [67]. We want to select $K$ candidate locations and create the connected graph $G_{1}$ and maximize the coverage. The main idea to maintain the graph connectivity is stated as follows: if a candidate location is selected, it injects 1 unit of flow to the network, and that flow can move from one node to another node if and only if those two nodes can see each other and are both selected. Up to now, we have $K$ units of flow inside the network and we force the entering flow to a node to be equal to the leaving flow from it. That is we force the flow conservation in the network. Then, if we allow the existence of only one sink node, which expels $K$ units of flow from the network, the resulting graph becomes connected. Because there must be a path between each node and the sink node, all nodes are connected to the sink and are therefore connected to any other node in the network.

To summarize the $G_{0}$ information, we create its adjacency matrix referred to as $A=\left\{a_{i j}\right\}_{n \times n}$, where $a_{i j}$ is 1 if and only if there is an edge between nodes $i$ and $j$, also one should note that in matrix $A$ the main diameter is 0 . To avoid negative flow between the edges, we create a directional graph out of $G_{0}$ where there are two links between each pair of nodes and we create the flow variable matrix $X=\left\{x_{i j}\right\}_{n \times n}$, 


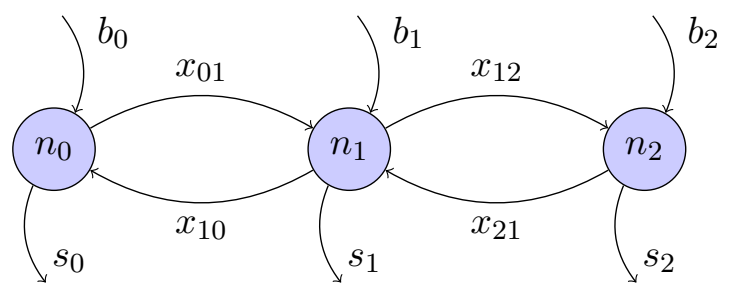

Figure 5.3: An example of the flow diagram in a three node network. The flow conservation assumption applies to all of the nodes in the graph, for $n_{1}$ it becoms as follows: $b_{1}+x_{01}+x_{21}-x_{10}-x_{12}=K s_{1}$, where $K$ is the number of candidate locations we want to select.

where the variable $x_{i j}$ represents the amount of flow traveling from node $i$ to node $j$. If a candidate location is selected, it is considered as a source node and it injects 1 unit of flow to the network, to find out which node is a source, we add a phantom link entering each candidate location and create the binary variable $B=\left\{b_{i}\right\}_{1 \times n}$, where $b_{i}$ is 1 if and only if node $i$ is selected. We take the same approach for the sink nodes and add a phantom link leaving each node so as to create the binary variable $S=\left\{s_{i}\right\}_{1 \times n}$, where $s_{i}$ is 1 if node $i$ is the network's sink node. Fig. 5.3 shows the flow diagram of a three-node network, and the formal statement of the optimization 
problem is as follows:

$$
\begin{array}{llr}
\underset{p, x, b, s}{\operatorname{maximize}} & \sum_{j=1}^{m} p_{j}, & \\
\text { subject to } & \sum_{i=1}^{n} c_{i j} b_{i} \geq p_{j}, & \\
& \sum_{i=1}^{n} b_{i}=K,\{1, \ldots, m\}, \\
& \sum_{i=1}^{n} s_{i}=1, & \\
& \sum_{j=0}^{n}\left(x_{j i}-x_{i j}\right)+b_{i}=K s_{i}, & \\
& x_{i j} \leq K b_{i}, K b_{j}, K a_{i j}, & i, j \in\{1, \ldots, n\}, \ldots, n\}, \\
& p_{j} \in\{0,1\}, & \\
& b_{i} \in\{0,1\}, \quad s_{i} \in\{0,1\}, & i \in\{1, \ldots, m\}, \\
& 0 \leq x_{i j} \leq K, & i, j \in\{1, \ldots, n\},
\end{array}
$$

where binary variable $P=\left\{p_{i}\right\}_{1 \times m}$ represents the points state. That is $p_{i}$ is 1 if and only if point $i$ is covered. The goal is to maximize the coverage, so the objective function is as 5.2.1. If a point is covered, at least one candidate location covering that point should be selected, resulting in 5.2.2. Also, there are $K$ selected nodes in total, so we have 5.2.3.

To ensure connectivity, we must only use one sink node, so we have the constraint 5.2.4. The flow conservation assumption forces the net flow entering and leaving a node to be zero, resulting in constraints 5.2.5. Finally, if $a_{i j}$ in the adjacency matrix is zero, or the node $i$ or the node $j$ is not selected, no flow can transfer 
between nodes $i$ and $j$, resulting in constraints 5.2.6.

\subsubsection{Greedy Approach}

In a real map scenario, we have a large number of candidate locations and the problem gets very large easily. Moreover, connectivity constraint is hard to achieve as it adds $n^{2}$ more variables to the formulation (variables $x$ in 5.2.1). As a result, the computational cost and time increases rapidly and, in certain cases, might explode.

To have an approximation for larger and more complicated maps, we introduce a greedy approach which scales much easier. We divide the problem into smaller steps, and in each step select the best. The main idea is stated as follows: in each step, select the candidate location which covers the largest number of points and sees at

least one candidate location which has already been selected. If we want to select $K$ ABSs, then the greedy method is formally stated as follows:

1. Select the node with the highest coverage.

2. Remove all covered points.

3. Assign $K \leftarrow K-1$.

4. If $K$ is zero, end.

5. Select the node with the highest coverage which sees at least one selected node which has already been selected.

6. Go to the step 2 .

\subsubsection{Upper Bound on Coverage}

To find out how many more BSs required when we force connectivity, we remove constraints (5.2.4), (5.2.5) and (5.2.6) and run the optimization. Also, we remove 
integer constraints to find an upper bound on the optimal coverage:

$$
\begin{array}{ll}
\underset{p, b}{\operatorname{maximize}} & \sum_{j=1}^{m} p_{j}, \\
\text { subject to } & \sum_{i=1}^{n} c_{i j} b_{i} \geq p_{j}, \quad j \in\{1, \ldots, m\}, \\
& \sum_{i=1}^{n} b_{i}=K .
\end{array}
$$

This formulation is a simple LP and is solvable in polynomial time. The bound is useful to analyze the greedy performance in case MILP is too costly.

\subsection{Minimizing the Number of Gateways and Load Balancing}

After selecting the ABS locations, we want to bring fiber (or any wired connection), to only a few of them and connect the rest of the ABSs via single or multiple-hop wireless links. This is mainly due to the high cost of installing fiber connections or using the currently deployed infrastructure.

Our primary goal in this section is to have minimmum gateway density. However, for a given gateway density, there can be different places for gateways as well as different routs for data flow to reach the core network. If we only consider gateway density in our planner, it is highly probable to have several gateways collecting most of the network load while there are many gateways collecting little of the network's load. Therefore, it is necessary to have a smart approach to avoid this issue, particularly for a network with greater number of allowable hops. Also, the network should be routed 
in a way to minimize the average number of hops needed for reaching the core of the network. Fewer hops lead to more reliability and smaller latencies in the network.

In this study, we consider a tree-topology backhaul network, where each ABS can forward its data to only one other ABS or gateway. To face the issues mentioned in above paragraph, we design a MILP such that the load is balanced throughout the network.

The MILP formulation considers the following objectives:

1. Minimize the gateway density.

2. Minimize the maximum capacity required.

3. Minimize the average number of hops.

Clearly, there is a trade-off between these demands. For example, if there are no constraints on the number of hops and the gateways capacity, then only 1 gateway would be enough for the entire network and, if capacity forces each gateway to serve only on cell's users, then all the ABSs should be upgraded to a gateway. However, as the limitations on the gateways capacity and the number of wireless hops is not well investigated for $5 \mathrm{G}$ networks [68], we give the priority to the gateway density and then we try to balance the network load. We strongly limit the number of allowable hops to be one or two; however, the formulation is well-justified for greater numbers 
of hops as well. The MILP formulation is as follows:

$$
\begin{array}{ll}
\underset{g, x, s, l}{\operatorname{minimize}} & c_{1} \sum_{i=1}^{k} g_{i}+c_{2} s_{\max }+c_{3} \sum_{i=1}^{k} \sum_{j=1}^{k} x_{i j}, \\
\text { subject to } & \sum_{i=1}^{k} u_{i j}(h) g_{i} \geq 1, \quad j \in K, \\
& s_{\max } \geq s_{i}, \quad i \in K, \\
& \sum_{i=1}^{k}\left(x_{j i}-x_{i j}\right)+1=s_{i}, \quad i \in K, \\
& x_{i j} \leq a_{i j}, \quad i \in K, j \in K, \\
& \sum_{i=1}^{k} l_{i j} \leq 1, \quad j \in K, \\
& x_{i j} \leq k l_{i j}, k\left(1-g_{i}\right) \quad i \in K, j \in K, \\
& l, g \in\{0,1\}, \\
& 0 \leq x, s, s_{\max } \leq k, \\
& K=\{1, \ldots, k\},
\end{array}
$$

where $c_{1}, c_{2}$, and $c_{3}$ are tuning parameters for the trade-off between minimizing the gateway density $\left(\sum_{i} g_{i}\right)$, the required maximum capacity $\left(s_{\max }\right)$, and the average number of hops (indirectly by $\sum_{i} \sum_{j} x_{i j}$ ), and $u$ is a binary matrix with $u_{i j}=1$ if and only if ABSs $i$ and $j$ can connect to each other within $h$ hops, where $h$ is the maximum number of allowable hops in the network. The binary decision variable $l$ and the continuous variable $x$ manage the flow of the network.

Constraint (5.4.2) states that each ABS should be reachable from one path within at most $h$ hops from a gateway. Constraint (5.4.3) forces all gateways' capacity to 
be less than $s_{\max }$ and therefore minimizes the maximum capacity. Constraint (5.4.4) is the network flow conservation assumption, where each ABS injects exactly 1 unit of flow to the network. Constraint (5.4.5) ensures the flow to move between adjacent ABSs. Finally, constraints (5.4.6) and (5.4.7) make the tree topology by forcing to have at most 1 exiting flow from each ABS and no existing flow from gateways $\left(x_{i j} \leq k\left(1-g_{i}\right)\right)$. 


\subsection{Simulations and Results}

We run the planner over 12 maps of Manhattan Island and 12 maps of central Paris, centered at $\left(73.9865^{\circ} \mathrm{W}, 40.7468^{\circ} \mathrm{N}\right)$ and $\left(2.334^{\circ} \mathrm{E}, 48.8600^{\circ} \mathrm{N}\right)$, respectively. Each map is $1 \mathrm{~km}^{2}$ wide and we consider the inner $0.36 \mathrm{~km}^{2}$ area to reduce the edge effect. First, we place many candidate locations, as described in Section 4.3, to find potential ABS sites. Second, we distribute grid points in the outdoor area with each point distance to its four neighbors set to $10 \mathrm{~m}$. This distance is fairly small to include almost all the outdoor area in the map. Smaller distances give us the same results, so we picked $10 \mathrm{~m}$ as a good approximation for outdoor area. Then we run the algorithm in 5.3 to select $K$ BSs and maximize the coverage area while the selected ABSs create a connected graph $G_{1}$. After finding the ABSs, we want to select the gateway places and we run the algorithm in 5.4 to minimize the total number of gateways. Although we solve the problem for different numbers of allowable hops, when the number of hops is increased one should consider the capacity limitations, latency, and reliability requirements of the wireless network. Therefore, we focus more on single-hop networks, but to give an idea of the change in gateway densities we run the algorithm for other numbers of hops as well.

In Section 5.5.1, we show the result of network planning on different real maps from Manhattan and Paris. Then, in Section 5.5.2, we provide some useful information about the possible ABSs and gateways densities for different LOS coverage ratios. Finally, in Section 5.5.3, we calculate the empirical distribution of bakchual link distances to give an impression of the possible LOS link distances in real maps. 


\subsubsection{Planned Networks}

Network planning results are shown in following figures. Clearly, for the larger allowable number of hops, the map size becomes critical, for example, if we want to plan a network with 3 number of hops, each map side length should at least be $3 \times 200 \times 2 \mathrm{~m}$ to fairly include the benefits of the wireless backhauling. Because, if we have 2 wireless backhaul, then each gateway can serve a user up to a maximum of $600 \mathrm{~m}$ away from itself.

\subsubsection{ABS and Gateway Densities}

Finding the number of ABSs and gateways required for outdoor LOS coverage is actually the main contribution of this work. Here, we give practical information regarding the possible densities. To be close to possible real scenarios, we found our results using real maps of two dense urban areas. Fig. 5.7 summarizes the discussion.

Different BS densities are required for different cities, which indicates the effect of the city geometry on the mmWave networks. As coverage area is LOS, the shape of a cell is highly affected by the buildings and street layouts, which shows its direct influence on the densities. Narrow bending streets and randomly shaped buildings reduce the chance of longe LOS link connection. As a result, it increases the BSs density.

Also, Fig. 5.7 includes the result of the greedy method and bound, which indicates that connectivity constraint affects the densities slightly and the bound is tight. Also, the greedy method performs well, as it requires at most 10 more ABSs per $\mathrm{km}^{2}$ to cover the area. We find 55 and 70 ABSs density as well as 18 and 23 gateways density 


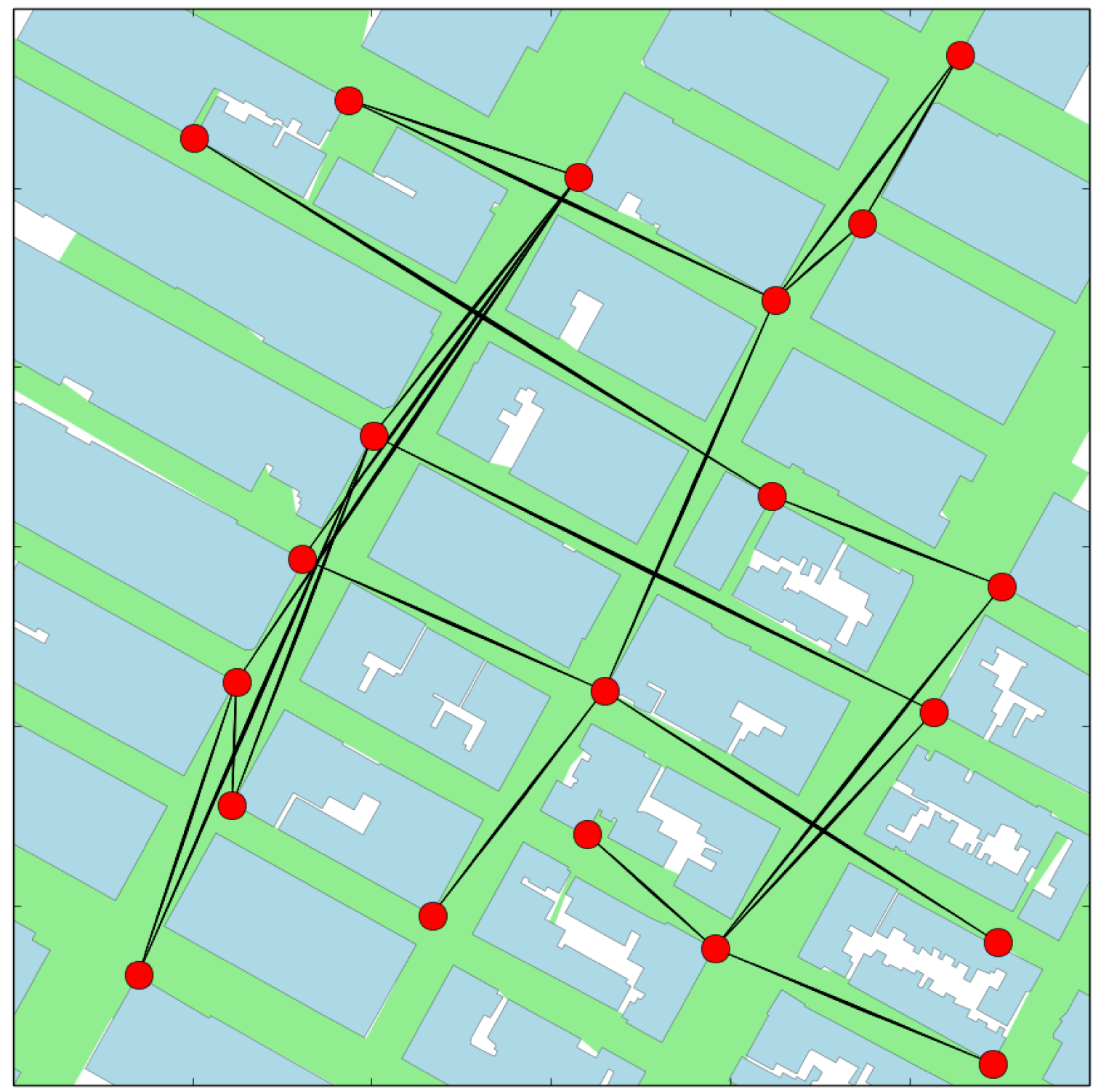

Figure 5.4: Result of maximum coverage problem with connectivity. Access base stations are shown with red circles, possible wireless backhaul links with black lines (representing the first Fresnel zone diamond approximation), and total LOS coverage area is shown with green. As can be seen, there are some places which are not covered, which is due to the fact that even when all candidate locations are selected there are some areas which are not covered. The places are found by running the algorithm in Section 5.3 over a $600 \mathrm{~m} \times 600 \mathrm{~m}$ area, with $K$ (number of access base stations) set to 20 , and the total coverage is around 92 percent of the outdoor area. 


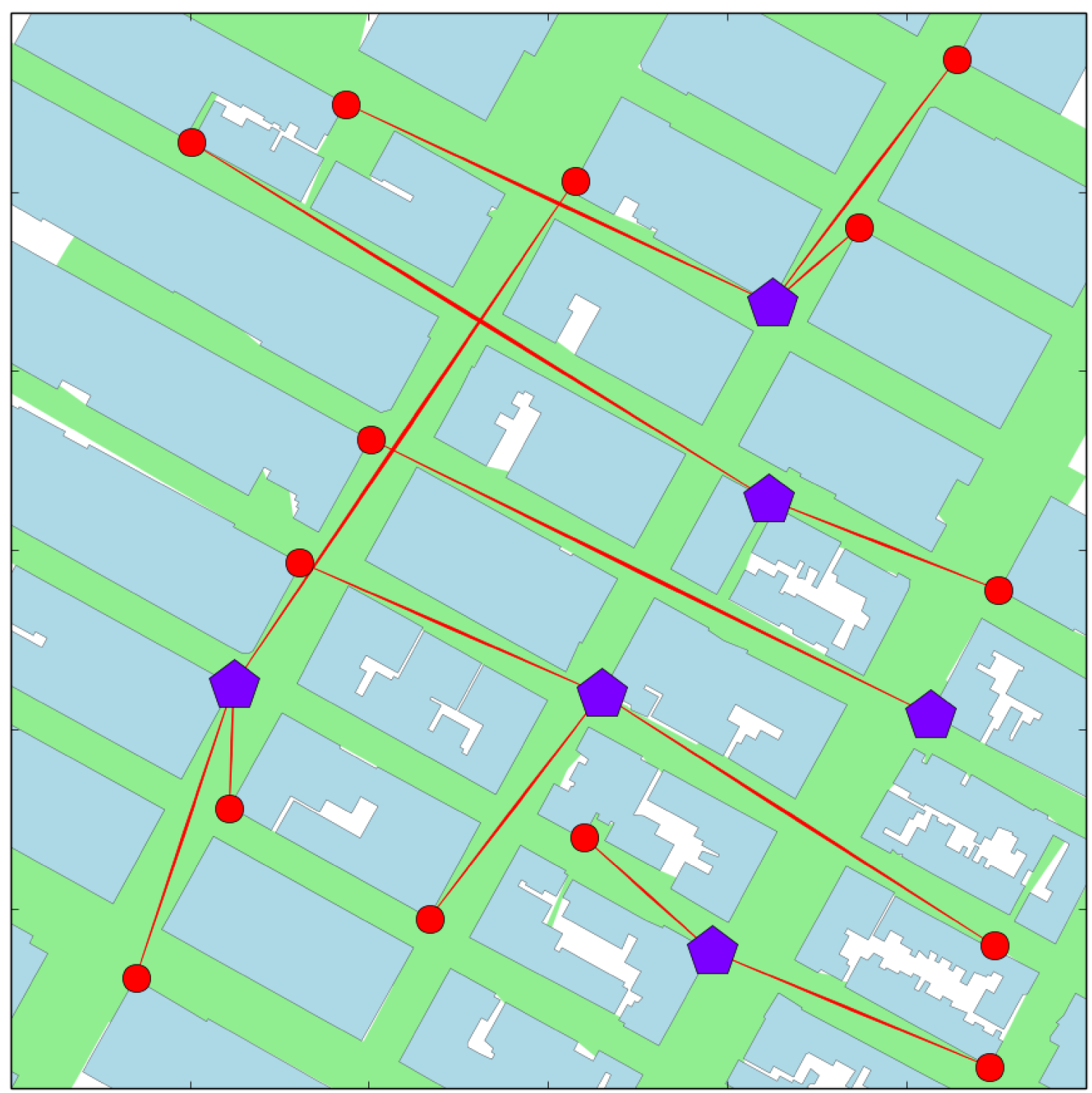

Figure 5.5: Minimizing the number of gateways. Result of the algorithm in Section 5.4 with the allowable number of hops set to 1 (single-hop network). Gateways are shown with purple pentagons, access base stations are shown with red circles, and the total line of sight coverage area is represented in green. In this particular map we need only 7 gateways to connect 20 base stations to the core network. 


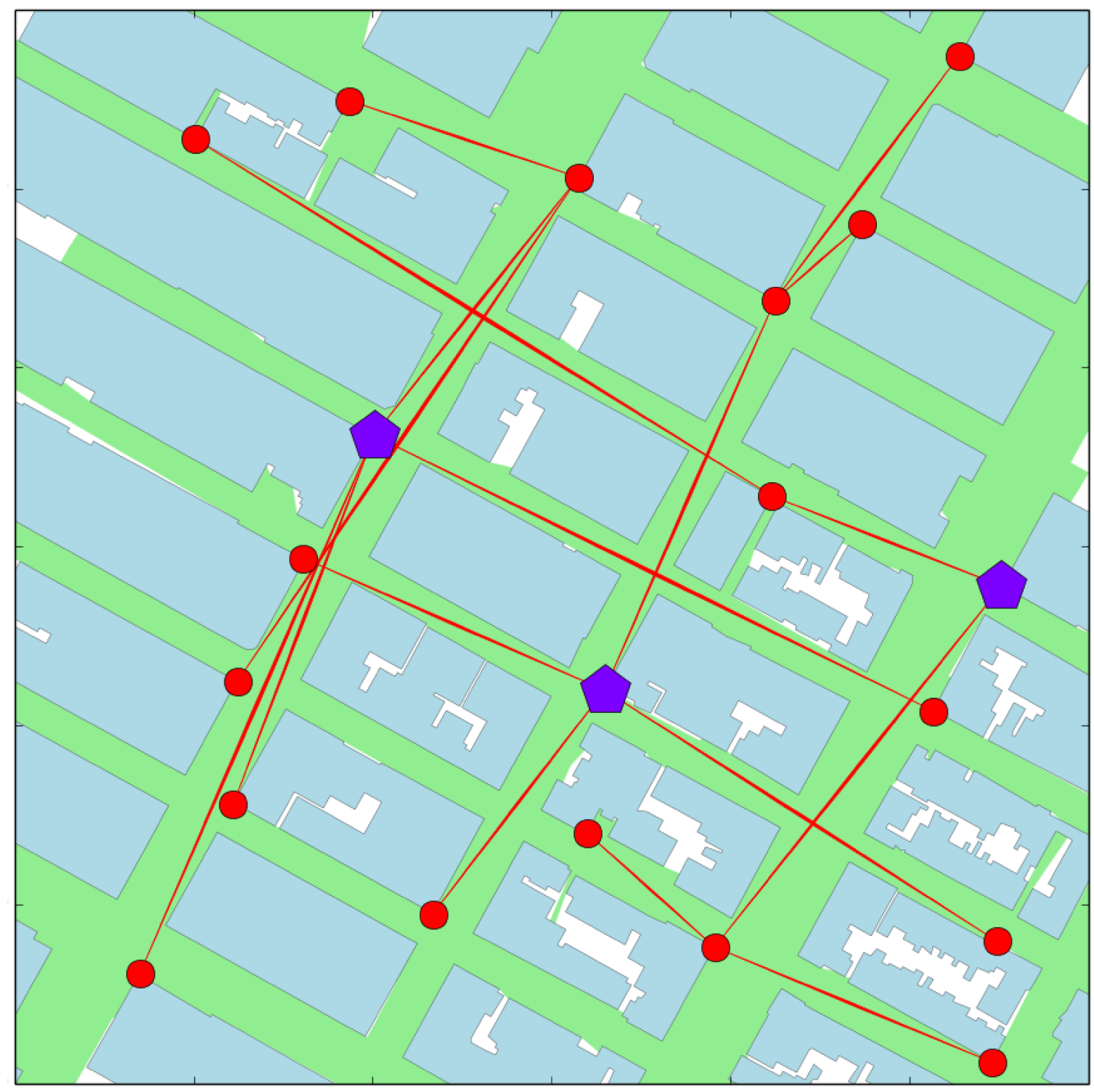

Figure 5.6: Minimizing the number of gateways. Result of the algorithm in Section 5.4 with allowable number of hops set to 2 . We solve the optimization problem to find the gateway locations and we create a tree-topology backhaul network. As can be seen, we can benefit a lot by increasing the number of hops, in this map we can connect a $600 \mathrm{~m} \times 600 \mathrm{~m}$ area with 3 gateways and $20 \mathrm{ABSs}$. Also, the network load is balanced over these GWs such that the maximum load is $7 \mathrm{ABSs}$ per gateway. 




ABSs and gateways densities per $\mathrm{km}^{2}$

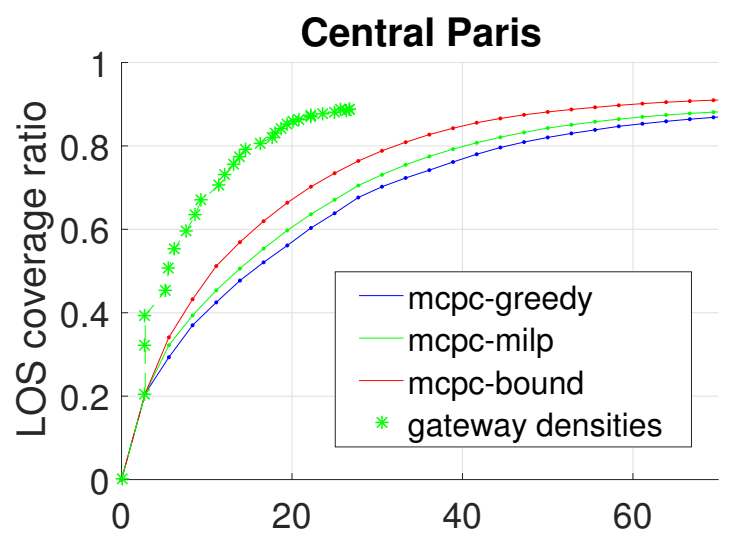

ABSs and gateways densities per $\mathrm{km}^{2}$

Figure 5.7: Access base stations and gateway densities for different line of sight coverages. We do not cover more than 92 percent, even if we increase the ABS density, as there are some places which do not have line of sight connection with any candidate locations.

to cover around 90 percent outdoor area in Manhattan and Paris, respectively.

\subsubsection{Probability Distribution of Links Distance}

Finally, Fig. 5.8 shows the empirical cumulative distribution function (CDF) of backhaul link distances. The difference between Manhattan and Paris emphasizes the effect of city layout on mmWave networks. Bending streets in Paris reduce the existence possibility of long-distance backhaul links.

\subsubsection{Load Balancing}

The average number of ABSs connected to a GW in one-and two-hop networks equals $55 / 20=2.75$ and $55 / 8=6.89$ (ABSs divided by GWs densities for 90\% coverage), respectively. As a result of the load balancing described in Section 5.4, more than $92 \%$ of these GWs serve less than 3 (and 7) ABSs for one-hop (and two-hop) networks, respectively. 




Figure 5.8: Emperical cumulative distribution function of link distances. Data suggest a uniform distribution. Also, the probability of having links longer than $300 \mathrm{~m}$ is less than 20 percent.

Also, we observe the average number of hops from user to GW is 1.68 (and 2.23) when one-hop (and two-hop) wireless backhauling is allowed, respectively. However, in two-hop networks, the GW density is decreased to less than half of the one-hop networks. 


\section{Chapter 6}

\section{Conclusion and Future Work}

\subsection{Conclusion}

Network planning is an important part of any network design, particularly in mmWave networks where the propagation characteristics are strongly affected by the environment. In this thesis, we designed a network planner for the outdoor area coverage. We used optimization and computational geometry and developed an automatic network planner. We further estimated several network properties. As this work contains simulation results as one of its contributions, we modeled the network by taking real map data (building footprints) into account. We extracted building footprints of two major dense urban areas from available open source databases and planned the network over them. Furthermore, to make our results more representative, we ran our simulations on many maps. Our contributions can be summarized as follows:

- Developed an automatic network planner by modelling the mmWave BS coverage area to be LOS and inside the small $(200 \mathrm{~m})$ radius of the BS. We further chose the wall-mounted BSs as a possible option to plan the network (as the wall locations are readily available from open source map data). In our planner, we first placed many candidate locations on good sites along each side of 
a building. Second, we selected a small subset of the candidates to minimize the number of BSs while bringing coverage to a high percentage of the outdoor area.

- Estimated the BSs density in mmWave networks by using our network planner and estimated the number of BSs required for mmWave networks. BSs density is an important factor in network design, as it contributes to the interference strength in the network and, more importantly, the BSs density directly translates into network cost.

- Found the effect of city geometry on the mmWave networks. After planning the network, we found different BS densities for different cities. Specifically, we ran our simulations over many maps of Manhattan Island and central Paris and observed that in Paris we need a higher number of BSs. These differences are the result of the shapes of the streets and the buildings. That is to say, narrow bending streets in Paris reduces the chance of having a long-distance wireless LOS link.

- Approximated the art gallery problem with cooperative guards in an engineering context. In order to provide wireless mmWave LOS backhaul links between BSs, we planned the network such that BSs see each other. That is, the selected candidates create a connected graph. The coverage problem with BSs seeing each other is actually analogous to the art gallery problem with cooperative guards. We used the connected subgraph problem and formulated a mixed integer linear programming problem to solve it.

- Estimated the gateways density. After planning the BSs on a connected graph, we minimized the number of installed fiber connections in a single-hop wireless 
backhaul network. Reducing fiber connections in the network can contribute to significantly reducing the network cost.

- As expected for mmWave networks, coverage, not the interference is the limitting factor.

\subsection{Future Work}

In this thesis, we use the $200 \mathrm{~m}$ LOS channel model to find the coverage areas. However, there might be enough signal power to communicate in some NLOS areas. For example, the first reflected ray can in many cases be a strong signal where the outer shell of city buildings are mostly made out of glass or other reflecting materials. Moreover, this simplified coverage model is more suitable for an average researcher, where ray-tracing tools are not easily accessible, rather than an industrial network planner. This model catches the basic and important aspects of the mmWave propagation, however, for a practical network planner one should use ray-tracing tools to find a more justified coverage estimation.

In the wireless backhaul planning chapter, we limit our analysis to single-hop wireless backhauling. However, the backhauling can be more beneficial in a larger number of hops, as it further reduces the wired gateways density. One can study the latency, reliability, and capacity of the connections, and increase the number of hops. Also, the number of access BSs connected to a gateway can be a limiting factor in these networks. As such adding a constraint to this parameter might be useful to ensure capacity requirements. Also, by increasing the number of hops one should develop routing algorithms to find the best paths to backhaul the users' data. The optimization formulation can be adopted to include these new ideas.

Finally, we work with $1 \mathrm{~km} \times 1 \mathrm{~km}$ map tiles in this thesis. However, working with 
these maps have noticable edge effect on our results. To find more reliable simulation results, one would need to work with larger map tiles. To make this possible we would need to make our algorithm formulation computationally simpler (fewer variables and constraints) or develop better heuristics. 


\section{List of References}

[1] J. G. Andrews, S. Buzzi, W. Choi, S. V. Hanly, A. Lozano, A. C. Soong, and J. C. Zhang, "What will 5G be?," IEEE Journal on Selected Areas in Communications, vol. 32, pp. 1065-1082, June 2014.

[2] T. S. Rappaport, S. Sun, R. Mayzus, H. Zhao, Y. Azar, K. Wang, G. N. Wong, J. K. Schulz, M. Samimi, and F. Gutierrez, "Millimeter wave mobile communications for 5G cellular: It will work!," IEEE Access, vol. 1, pp. 335-349, May 2013.

[3] C. Dehos, J. L. Gonzalez, A. De Domenico, D. Ktenas, and L. Dussopt, "Millimeter-wave access and backhauling: the solution to the exponential data traffic increase in $5 \mathrm{G}$ mobile communications systems?" IEEE Communications Magazine, vol. 52, pp. 88-95, September 2014.

[4] P. Calégari, F. Guidec, and P. Kuonen, "Urban radio network planning for mobile phones," EPFL Supercomputing Review (series in Numerical Simulation for Science and Technology), pp. 4-10, November 1997.

[5] J. Wong, A. Mason, M. Neve, and K. Sowerby, "Base station placement in indoor wireless systems using binary integer programming," IEEE ProceedingsCommunications, vol. 153, pp. 771-778, October 2006.

[6] L. Pujji, K. Sowerby, and M. Neve, "Development of a hybrid algorithm for efficient optimisation of base station placement for indoor wireless communication systems.," Wireless Personal Communications, vol. 69, pp. 471-486, March 2013.

[7] N. Palizban, S. Szyszkowicz, and H. Yanikomeroglu, "Automation of millimeter wave network planning for outdoor coverage in dense urban areas using wallmounted base stations," IEEE Wireless Communications Letters, vol. 6, pp. 206209, April 2017. 
[8] P. Calégari, F. Guidec, P. Kuonen, and F. Nielsen, "Combinatorial optimization algorithms for radio network planning," Theoretical Computer Science, vol. 263, pp. 235-245, July 2001.

[9] T. Bai, R. Vaze, and R. W. Heath, "Analysis of blockage effects on urban cellular networks," IEEE Transactions on Wireless Communications, vol. 13, pp. 50705083, September 2014.

[10] T. Bai and R. W. Heath, "Coverage analysis for millimeter wave cellular networks with blockage effects," in IEEE Global Conference on Signal and Information Processing (GlobalSIP), pp. 727-730, Febuary 2013.

[11] W. Lu and M. di Renzo, "Stochastic geometry modeling of mmWave cellular networks: Analysis and experimental validation," in IEEE International Workshop on Measurements $\&$ Networking, pp. 1-4, October 2015.

[12] M. di Renzo, "Stochastic geometry modeling and analysis of multi-tier millimeter wave cellular networks," IEEE Transaction on Wireless Communications, vol. 14, pp. 5038-5057, September 2015.

[13] S. Szyszkowicz, A. Lou, and H. Yanikomeroglu, "Automated placement of individual millimeter-wave wall-mounted base stations for line-of-sight coverage of outdoor urban areas," IEEE Wireless Communications Letters, vol. 5, April 2016.

[14] A. Damnjanovic, J. Montojo, Y. Wei, T. Ji, T. Luo, M. Vajapeyam, T. Yoo, O. Song, and D. Malladi, "A survey on 3GPP heterogeneous networks," IEEE Wireless Communications, vol. 18, pp. 10-21, June 2011.

[15] B. Naeem, R. Ngah, and S. Z. M. Hashim, "Handovers in small cell based heterogeneous networks," in International Conference on Computing, Electronic and Electrical Engineering (ICE Cube), pp. 268-271, April 2016.

[16] H. Zhang, C. Jiang, J. Cheng, and V. C. M. Leung, "Cooperative interference mitigation and handover management for heterogeneous cloud small cell networks," IEEE Wireless Communications, vol. 22, pp. 92-99, June 2015.

[17] G. Godor, Z. Jako, A. Knapp, and S. Imre, "A survey of handover management in LTE-based multi-tier femtocell networks: Requirements, challenges and solutions," Computer Networks, vol. 76, pp. 17-41, November 2014. 
[18] S. S. Sehra, J. Singh, and H. S. Rai, "A systematic study of OpenStreetMap data quality assessment," in Information Technology: New Generations International Conference (ITNG), pp. 377-381, April 2014.

[19] M. N. Kulkarni, S. Singh, and J. G. Andrews, "Coverage and rate trends in dense urban mmWave cellular networks," in IEEE Global Communications Conference, pp. 3809-3814, December 2014.

[20] C. V. N. Index, "Cisco visual networking index: forecast and methodology, 20152020," Tech. Rep, 2015.

[21] E. Hossain and M. Hasan, "5G cellular: key enabling technologies and research challenges," IEEE Instrumentation \& Measurement Magazine, vol. 18, pp. 11-21, June 2015.

[22] J. G. Andrews, T. Bai, M. N. Kulkarni, A. Alkhateeb, A. K. Gupta, and R. W. Heath, "Modeling and analyzing millimeter wave cellular systems," IEEE Transactions on Communications, vol. 65, pp. 403-430, January 2017.

[23] B. Bangerter, S. Talwar, R. Arefi, and K. Stewart, "Networks and devices for the 5G era," IEEE Communications Magazine, vol. 52, pp. 90-96, Febuary 2014.

[24] W. Roh, J.-Y. Seol, J. Park, B. Lee, J. Lee, Y. Kim, J. Cho, K. Cheun, and F. Aryanfar, "Millimeter-wave beamforming as an enabling technology for 5G cellular communications: theoretical feasibility and prototype results," IEEE Communications Magazine, vol. 52, pp. 106-113, Febuary 2014.

[25] F. W. Vook, A. Ghosh, and T. A. Thomas, "MIMO and beamforming solutions for 5G technology," in IEEE MTT-S International Microwave Symposium (IMS), pp. 1-4, July 2014.

[26] M. R. Akdeniz, Y. Liu, M. K. Samimi, S. Sun, S. Rangan, T. S. Rappaport, and E. Erkip, "Millimeter wave channel modeling and cellular capacity evaluation," IEEE Journal on Selected Areas in Communications, vol. 32, pp. 1164-1179, June 2014.

[27] T. Imai, K. Kitao, N. Tran, N. Omaki, Y. Okumura, and K. Nishimori, "Outdoorto-indoor path loss modeling for 0.8 to $37 \mathrm{GHz}$ band," in IEEE European Conference on Antennas and Propagation (EuCAP), pp. 1-4, June 2016. 
[28] I. Rodriguez, H. C. Nguyen, N. T. Jorgensen, T. B. Sorensen, and P. Mogensen, "Radio propagation into modern buildings: Attenuation measurements in the range from $800 \mathrm{MHz}$ to $18 \mathrm{GHz}$," in IEEE Vehicular Technology Conference (VTC Fall), pp. 1-5, December 2014.

[29] R. Baldemair, T. Irnich, K. Balachandran, E. Dahlman, G. Mildh, Y. Selén, S. Parkvall, M. Meyer, and A. Osseiran, "Ultra-dense networks in millimeterwave frequencies," IEEE Communications Magazine, vol. 53, pp. 202-208, January 2015.

[30] N. Bhushan, J. Li, D. Malladi, R. Gilmore, D. Brenner, A. Damnjanovic, R. Sukhavasi, C. Patel, and S. Geirhofer, "Network densification: the dominant theme for wireless evolution into 5G," IEEE Communications Magazine, vol. 52, pp. 82-89, February 2014.

[31] B. Ayvazian, "True non-line-of-sight is critical for outdoor urban small cell backhaul," White Paper in Heavy Reading, January 2014.

[32] M. Coldrey, J.-E. Berg, L. Manholm, C. Larsson, and J. Hansryd, "Non-line-ofsight small cell backhauling using microwave technology," IEEE Communications Magazine, vol. 51, pp. 78-84, September 2013.

[33] S. Chia, M. Gasparroni, and P. Brick, "The next challenge for cellular networks: backhaul," IEEE Microwave Magazine, vol. 10, pp. 54-66, August 2009.

[34] W. Feng, Y. Li, D. Jin, L. Su, and S. Chen, "Millimetre-wave backhaul for 5G networks: Challenges and solutions," Sensors, vol. 16, pp. 892-909, June 2016.

[35] M. Talau, E. C. Wille, and H. S. Lopes, "Solving the base station placement problem by means of swarm intelligence," in IEEE Symposium on Computational Intelligence for Communication Systems and Networks (CIComms), pp. 39-44, August 2013.

[36] OFCOM, "Sitefinder database." http://web.archive.org/web/ 20080207010024/http://www . 808multimedia.com/winnt/kernel.htm.

[37] O. S. B. mapping agency, "OS open data." https://www.ordnancesurvey.co . uk/opendatadown-load/products.html.

[38] S. Coast, "Openstreetmaps." http://www.openstreetmap.org. 
[39] M. Helbich, C. Amelunxen, P. Neis, and A. Zipf, "Comparative spatial analysis of positional accuracy of openstreetmap and proprietary geodata," Proceedings of GI_Forum, pp. 24-33, 2012.

[40] M. Gapeyenko, A. Samuylov, M. Gerasimenko, D. Moltchanov, S. Singh, E. Aryafar, S. p. Yeh, N. Himayat, S. Andreev, and Y. Koucheryavy, "Analysis of human-body blockage in urban millimeter-wave cellular communications," in IEEE International Conference on Communications (ICC), pp. 1-7, May 2016.

[41] F. Bungiu, M. Hemmer, J. Hershberger, K. Huang, and A. Kröller, "Efficient computation of visibility polygons," arXiv preprint arXiv:1403.3905, March 2014.

[42] S. K. Ghosh, Visibility Algorithms in The Plane. Cambridge University Press, 2007.

[43] K. J. Obermeyer and Contributors, "The VisiLibity library." http://www.VisiLibity.org, 2008. Release 1.

[44] J. Urrutia et al., "Art gallery and illumination problems," Handbook of Computational Geometry, vol. 1, pp. 973-1027, April 2000.

[45] V. Chvatal, "A combinatorial theorem in plane geometry," Journal of Combinatorial Theory, Series B, vol. 18, pp. 39-41, Febuary 1975.

[46] V. Pinciu, "A coloring algorithm for finding connected guards in art galleries," in Discrete Mathematics and Theoretical Computer Science, pp. 257-264, Springer, July 2003.

[47] T. Michael and V. Pinciu, "Art gallery theorems for guarded guards," Computational Geometry, vol. 26, pp. 247-258, November 2003.

[48] G. Hernandez-Penalver, "Controlling guards.," in The Sixth Canadian Conference on Computational Geometry, pp. 387-392, 1994.

[49] D. C. Tozoni, P. J. de Rezende, and C. C. de Souza, "A practical iterative algorithm for the art gallery problem using integer linear programming," Optimization Online, October 2013.

[50] S. S. Ray, Graph Theory with Algorithms and Its Applications: In Applied Science and Technology. Springer Science \& Business Media, 2012. 
[51] D. B. West et al., Introduction to Graph Theory. Prentice Hall Upper Saddle River, 2001.

[52] M. Fiedler, "Algebraic connectivity of graphs," Czechoslovak Mathematical Journal, vol. 23, no. 2, pp. 298-305, 1973.

[53] A. Berman and X.-D. Zhang, "Lower bounds for the eigenvalues of laplacian matrices," Linear Algebra and its Applications, vol. 316, pp. 13-20, Febuary 2000.

[54] A. Torgasev and M. Petrovic, "Lower bounds of the laplacian graph eigenvalues," Indagationes Mathematicae, vol. 15, pp. 589-593, December 2004.

[55] E. Alvarez-Miranda, I. Ljubic, and P. Mutzel, "The maximum weight connected subgraph problem," in Facets of Combinatorial Optimization, pp. 245270, Springer, September 2013.

[56] S. Boyd and L. Vandenberghe, Convex Optimization. Cambridge University Press, 2004.

[57] W. L. Winston, M. Venkataramanan, and J. B. Goldberg, Introduction to Mathematical Programming. Thomson/Brooks/Cole Duxbury; Pacific Grove, CA, 2003.

[58] R. Hofman, "Why linear programming cannot solve large instances of npcomplete problems in polynomial time," arXiv preprint cs/0611008, November 2006.

[59] F. Khan and Z. Pi, "mmWave mobile broadband MMB: Unleashing the 3-300 GHz spectrum," in IEEE Sarnoff Symposium, pp. 1-6, May 2011.

[60] D. S. Hochbaum and A. Pathria, "Analysis of the greedy approach in problems of maximum k-coverage," Naval Research Logistics, vol. 45, pp. 615-627, September 1998.

[61] U. Feige, "A threshold of ln n for approximating set cover," Journal of the ACM, vol. 45, pp. 634-652, July 1998.

[62] R. L. Church and R. R. Davis, "The fixed charge maximal covering location problem," Papers in Regional Science, vol. 71, pp. 199-215, July 1992. 
[63] T. S. Rappaport, F. Gutierrez, E. Ben-Dor, J. N. Murdock, Y. Qiao, and J. I. Tamir, "Broadband millimeter-wave propagation measurements and models using adaptive-beam antennas for outdoor urban cellular communications," IEEE Transactions on Antennas Propagation, vol. 61, pp. 1850-1859, December 2013.

[64] T. S. Rappaport, G. R. MacCartney, M. K. Samimi, and S. Sun, "Wideband millimeter-wave propagation measurements and channel models for future wireless communication system design," IEEE Transactions on Communications, vol. 63, pp. 3029-3056, May 2015.

[65] M. Rebato, M. Mezzavilla, S. Rangan, F. Boccardi, and M. Zorzi, "Understanding noise and interference regimes in 5G millimeter-wave cellular networks," in IEEE European Wireless Conference, pp. 1-5, May 2016.

[66] B.-C. Liaw, N.-F. Huang, and R. C. Lee, "The minimum cooperative guards problem on k-spiral polygons," in The Fifth Canadian Conference on Computational Geometry, pp. 97-102, August 1993.

[67] J. Conrad, C. P. Gomes, W.-J. van Hoeve, A. Sabharwal, and J. Suter, "Connections in networks: Hardness of feasibility versus optimality," in International Conference on Integration of Artificial Intelligence (AI) and Operations Research (OR) Techniques in Constraint Programming, pp. 16-28, May 2007.

[68] X. Ge, L. Pan, S. Tu, H. H. Chen, and C. X. Wang, "Wireless backhaul capacity of 5G ultra-dense cellular networks," in IEEE 84th Vehicular Technology Conference (VTC-Fall), pp. 1-6, Sept. 2016. 